\title{
Induced Convolution Operator Norms of Linear Dynamical Systems*
}

\author{
VijaySekhar Chellaboina,$^{\dagger}$ Wassim M. Haddad ${ }^{\ddagger}$ \\ Dennis S. Bernstein, $\S$ and David A. Wilson"
}

\begin{abstract}
In this paper we develop explicit formulas for induced convolution operator norms and their bounds. These results generalize established induced operator norms for linear dynamical systems with various classes of input-output signal pairs.
\end{abstract}

Key words. Convolution operators, Hankel operators, Induced norms, Mixedinduced norms, Bounded energy signals, Bounded amplitude signals, $\mathrm{H}_{\infty}$ norm, $\mathrm{L}_{1}$ norm.

\section{Introduction}

In this paper we consider the dynamical system

$$
\begin{aligned}
& \dot{x}(t)=A x(t)+B u(t), \quad x(0)=0, \quad t \geq 0, \\
& y(t)=C x(t),
\end{aligned}
$$

where $x(t) \in \mathbb{R}^{n}, u(t) \in \mathbb{R}^{m}, y(t) \in \mathbb{R}^{l}, \quad t \in[0, \infty), A \in \mathbb{R}^{n \times n}$ is asymptotically stable, $B \in \mathbb{R}^{n \times m}$, and $C \in \mathbb{R}^{l \times n}$. Here, $u(\cdot)$ is an input signal belonging to the class $\mathrm{L}_{p}$ of input signals and $y(\cdot)$ is an output signal belonging to the class $\mathrm{L}_{r}$ of output signals, where the notation $\mathrm{L}_{p}$ denotes the set of functions in $\mathrm{L}_{p}$. In applications, (1) and (2) may denote a control system in closed-loop configuration where the objective is to determine the "size" of the output $y(\cdot)$ for a disturbance $u(\cdot)$. In this paper we develop explicit formulas for convolution operator norms

* Date received: April 1, 1999. Date revised: February 21, 2000. This research was supported in part by the National Science Foundation under Grant ECS-9496249 and the Air Force Office of Scientific Research under Grants F49620-001-0095 and F49620-98-1-0037.

$\dagger$ Mechanical and Aerospace Engineering, University of Missouri-Columbia, Columbia, Missouri 65211-2200, U.S.A. ChellaboinaV@missouri.edu.

$\ddagger$ School of Aerospace Engineering, Georgia Institute of Technology, Atlanta, Georgia 30332-0150, U.S.A.wm.haddad@aerospace.gatech.edu.

$\S$ Department of Aerospace Engineering, The University of Michigan, Ann Arbor, Michigan 481092140, U.S.A. dsbaero@umich.edu.

$\|$ Department of Electronic and Electrical Engineering, The University of Leeds, Leeds LS2 9JT, England. daw@elec-eng.leeds.ac.uk. 
and their bounds induced by various norms on several classes of input-output signal pairs. These results generalize established induced convolution operator norms for linear dynamical systems.

If the input-output signals are constrained to be finite energy signals so that $u, y \in \mathrm{L}_{2}$, then the equi-induced (that is, the domain and range spaces of the convolution operator are assigned the same temporal and spatial norms) signal norm is the $\mathrm{H}_{\infty}$ system norm $[\mathrm{F}],[\mathrm{Z}]$ given by

$$
\|\mathcal{G}\|_{(2,2),(2,2)} \triangleq \sup _{u(\cdot) \in \mathrm{L}_{2}} \frac{\|y\|_{2,2}}{\|u\|_{2,2}}=\sup _{\omega \in \mathbb{R}} \sigma_{\max }[H(\jmath \omega)],
$$

where the notation $\|\cdot\|_{p, q}$ denotes a signal norm with $p$ temporal norm and $q$ spatial norm, $\sigma_{\max }(\cdot)$ denotes the maximum singular value, $\mathcal{G}$ denotes the convolution operator of $(1),(2)$, and $H(s)=C(s I-A)^{-1} B$ is the corresponding transfer function. Hence, the $\mathrm{H}_{\infty}$ system norm captures the supremum system energy gain.

Alternatively, if the input-output signals are constrained to be bounded amplitude signals so that $u, y \in \mathrm{L}_{\infty}$, then the equi-induced signal norm

$$
\|\mathcal{G}\|_{(\infty, \infty),(\infty, \infty)} \triangleq \sup _{u(\cdot) \in \mathrm{L}_{\infty}} \frac{\|y\| \|_{\infty, \infty}}{\|u\| \|_{\infty, \infty}}
$$

is the $\mathrm{L}_{1}$ system norm ${ }^{1}[\mathrm{DD}],[\mathrm{V}]$. Thus, the $\mathrm{L}_{1}$ system norm captures the worstcase amplification from input disturbance signals to output signals, where the signal size is taken to be the supremum over time of the signal's peak value pointwise in time [DD], [V].

Mixed input-output signals have also been considered. For example, if $u \in \mathrm{L}_{2}$ and $y \in \mathrm{L}_{\infty}$, then the resulting induced operator norm is [W2]

$$
\|\mid \mathcal{G}\|_{(\infty, 2),(2,2)} \triangleq \sup _{u(\cdot) \in \mathrm{L}_{2}} \frac{\|y\|_{\infty, 2}}{\|u\|_{2,2}}=\lambda_{\max }\left(C Q C^{\mathrm{T}}\right),
$$

where $\lambda_{\max }(\cdot)$ denotes the maximum eigenvalue and $Q$ is the unique $n \times n$ nonnegative-definite solution to the Lyapunov equation

$$
0=A Q+Q A^{\mathrm{T}}+B B^{\mathrm{T}}
$$

Hence, $\||\mathcal{G}|\|_{(\infty, 2),(2,2)}$ provides a worst-case measure of amplitude errors due to finite energy input signals. Alternatively, if the input and output signal norms are chosen as $\||\cdot|\|_{2,2}$ and $\|\mid \cdot\| \|_{\infty, \infty}$, respectively, then the resulting induced operator norm is [W2]

$$
\|\mid \mathcal{G}\|_{(\infty, \infty),(2,2)} \triangleq \sup _{u(\cdot) \in \mathrm{L}_{2}} \frac{\|y \mid\| \|_{\infty, \infty}}{\|u\|_{2,2}}=\mathrm{d}_{\max }\left(C Q C^{\mathrm{T}}\right),
$$

where $\mathrm{d}_{\max }(\cdot)$ denotes the maximum diagonal element. Hence, $\|\mid \mathcal{G}\|_{(\infty, \infty),(2,2)}$ provides a worst-case peak excursion response due to finite energy disturbances.

\footnotetext{
${ }^{1}$ In the single-input/single-output case, it is well known that the induced norm (4) corresponds to the $\mathrm{L}_{1}$ norm of the impulse response matrix function [DD], [V].
} 
It is clear from the above discussion that operator norms induced by classes of input-output signal pairs can be used to capture disturbance rejection performance objectives for controlled dynamical systems. In particular, $\mathrm{H}_{\infty}$ control theory $[F],[Z]$ has been developed to address the problem of disturbance rejection for systems with bounded energy $\mathrm{L}_{2}$ signal norms on the disturbance and performance variables. Since the induced $\mathrm{H}_{\infty}$ transfer function norm (3) corresponds to the worst-case disturbance attenuation, for systems with $\mathrm{L}_{2}$ disturbances which possess significant power within arbitrarily small bandwidths, $\mathrm{H}_{\infty}$ theory is clearly appropriate. Alternatively, to address pointwise in time worst-case peak amplitude response due to bounded amplitude persistent $\mathrm{L}_{\infty}$ disturbances, $\mathrm{L}_{1}$ theory is appropriate [DD], [V]. The problem of finding a stabilizing controller such that the closed-loop system gain from $\||\cdot|\|_{2,2}$ to $\left\||\cdot \||_{\infty, q}\right.$, where $q=2$ or $\infty$, is below a specified level is solved in $[\mathrm{R}]$ and [WNH]. In addition to the disturbance rejection problem, another application of induced operator norms is the problem of actuator amplitude and rate saturation [DEBW], [CH2]. In particular, since the convolution operator norm $\|\mid \mathcal{G}\|_{(\infty, \infty),(2,2)}$ given by (7) captures the worst-case peak amplitude response due to finite energy disturbances, defining the output (performance) variables $y$ to correspond to the actuator amplitude and actuator rate signals, it follows that $\|\mid \mathcal{G}\|_{(\infty, \infty),(2,2)}$ bounds actuator amplitude and actuator rate excursion. Furthermore, since uncertain signals can also be used to model uncertainty in a system, the treatment of certain classes of uncertain disturbances also enable the development of controllers that are robust with respect to input-output uncertainty blocks [DD], [DV].

In the recent papers [W2] and [W3], Wilson developed explicit formulas for convolution operator norms induced by several classes of input-output signal pairs. In this paper we extend the results of [W2] and [W3] to a larger class of input-output signal pairs and provide explicit formulas for induced convolution operator norms and operator norm bounds for linear dynamical systems. These results generalize several well-known induced convolution operator norm results in the literature including results on $\mathrm{L}_{\infty}$ equi-induced norms ( $\mathrm{L}_{1}$ operator norms) and $\mathrm{L}_{1}$ equi-induced norms (resource norms). In cases where the induced convolution operator norm expressions are not finitely computable, we provide finitely computable norm bounds. Finally, since a single performance objective is seldom adequate for capturing multiple and often conflicting design objectives $[\mathrm{BH}]$, $[\mathrm{ZGBD}],[\mathrm{SHB}]$, induced convolution operator norms for mixed-norm linear dynamical systems are also developed.

\section{Notation}

$\begin{array}{ll}\mathbb{R}, \mathbb{R}^{m \times n} & \text { real numbers, } m \times n \text { real matrices } \\ x_{i} & i \text { th entry of vector } x \\ |x| & \text { vector whose } i \text { th element is }\left|x_{i}\right| \\ e_{i} & \text { vector with unity in } i \text { th position and zeros elsewhere } \\ A_{(i, j)} & (i, j) \text { th element of matrix } A \\ A \leq \leq B & A_{(i, j)} \leq B_{(i, j)} \text { for all } i \text { and } j, \text { where } A \text { and } B \text { are real matrices } \\ & \quad \text { with identical dimensions } \\ \operatorname{row}_{i}(A), \operatorname{col}_{i}(A) & i \text { th row of } A, i \text { th column of } A \\ A^{\mathrm{T}} & \text { transpose of } A\end{array}$




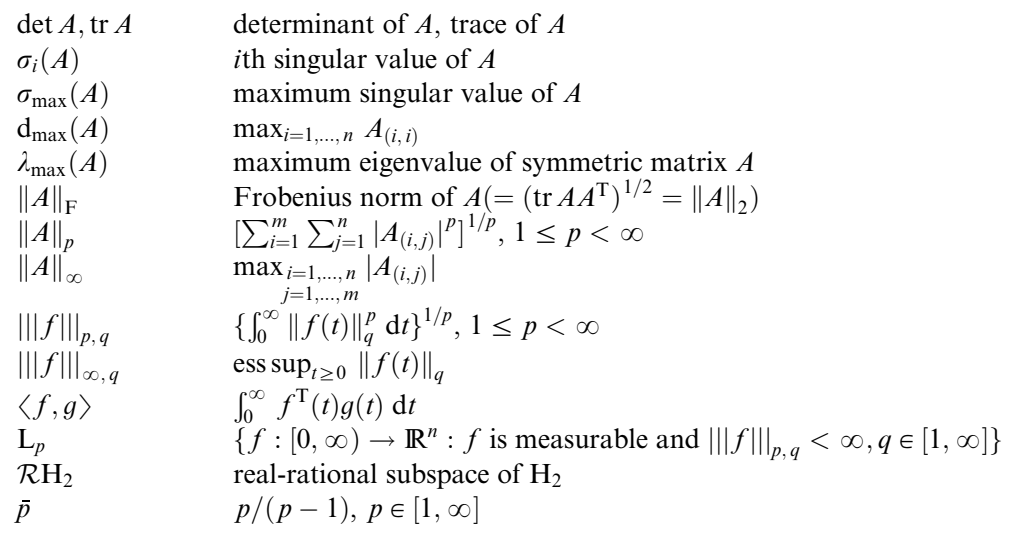

\section{Mathematical Preliminaries}

Let $\|\cdot\|^{\prime}$ and $\|\cdot\|^{\prime \prime}$ denote vector norms on $\mathbb{R}^{n}$ and $\mathbb{R}^{m}$, respectively, where $m, n \geq 1$. Then $\|\cdot\|: \mathbb{R}^{m \times n} \rightarrow \mathbb{R}$ defined by

$$
\|A\| \triangleq \max _{\|x\|^{\prime}=1}\|A x\|^{\prime \prime}
$$

is the matrix norm induced by $\|\cdot\|^{\prime}$ and $\|\cdot\|^{\prime \prime}$. If $\|\cdot\|^{\prime}=\|\cdot\|_{p}$ and $\|\cdot\|^{\prime \prime}=\|\cdot\|_{q}$, where $p, q \in[1, \infty]$, then the matrix norm on $\mathbb{R}^{m \times n}$ induced by $\|\cdot\|_{p}$ and $\|\cdot\|_{q}$ is denoted by $\|\cdot\|_{q, p}$. Let $\|\cdot\|$ denote a vector norm on $\mathbb{R}^{m}$. Then the dual norm $\|\cdot\|_{\mathrm{D}}$ of $\|\cdot\|$ is defined by

$$
\|y\|_{\mathrm{D}} \triangleq \max _{\|x\|=1}\left|y^{\mathrm{T}} x\right|
$$

where $y \in \mathbb{R}^{m}[\mathrm{SS}]$. Note that $\|\cdot\|_{\mathrm{DD}}=\|\cdot\|[\mathrm{SS}]$. Furthermore, if $p, q \in[1, \infty]$ satisfy $1 / p+1 / q=1$, then $\|\cdot\|_{p \mathrm{D}}=\|\cdot\|_{q}[\mathrm{SS}]$. For $p \in[1, \infty]$ we denote the conjugate variable $q \in[1, \infty]$ satisfying $1 / p+1 / q=1$ by $\bar{p}=p /(p-1)$.

Let $\|\cdot\|$ denote a vector norm on $\mathbb{R}^{n}$. Then $\|\cdot\|$ is absolute if $\|x\|=\||x|\|$ for all $x \in \mathbb{R}^{n}$. Furthermore, $\|\cdot\|$ is monotone if $\|x\| \leq\|y\|$ for all $x, y \in \mathbb{R}^{n}$ such that $|x| \leq \leq|y|$. Note that $\|\cdot\|$ is absolute if and only if $\|\cdot\|$ is monotone [HJ, p. 285].

Lemma 2.1. Let $p \in[1, \infty]$ and let $A \in \mathbb{R}^{m \times n}$. Then

$$
\begin{aligned}
\|A\|_{2,2} & =\sigma_{\max }(A), \\
\|A\|_{p, 1} & =\max _{i=1, \ldots, n}\left\|\operatorname{col}_{i}(A)\right\|_{p},
\end{aligned}
$$

and

$$
\|A\|_{\infty, p}=\max _{i=1, \ldots, m}\left\|\operatorname{row}_{i}(A)\right\|_{\bar{p}} .
$$

Proof. Expression (8) is standard; see [SS] for a proof. To show (9), note that, for all $x \in \mathbb{R}^{n}$,

$$
\|A x\|_{p}=\left\|\sum_{i=1}^{n} x_{i} \operatorname{col}_{i}(A)\right\|_{p} \leq \sum_{i=1}^{n}\left|x_{i}\right|\left\|\operatorname{col}_{i}(A)\right\|_{p} \leq \max _{i=1, \ldots, n}\left\|\operatorname{col}_{i}(A)\right\|\left\|_{p}\right\| x \|_{1}
$$


and hence $\|A\|_{p, 1} \leq \max _{i=1, \ldots, n}\left\|\operatorname{col}_{i}(A)\right\|_{p}$. Next, let $j \in\{1, \ldots, n\}$ be such that $\left\|\operatorname{col}_{j}(A)\right\|_{p}=\max _{i=1, \ldots, n}\left\|\operatorname{col}_{i}(A)\right\|_{p}$. Now, since $\left\|e_{j}\right\|_{1}=1$, it follows that $\left\|A e_{j}\right\|_{p}=$ $\left\|\operatorname{col}_{j}(A)\right\|_{p}$ which implies $\|A\|_{p, 1} \geq \max _{i=1, \ldots, n}\left\|\operatorname{col}_{i}(A)\right\|_{p}$ and hence (9) holds.

Finally, to show (10) note that, for all $x \in \mathbb{R}^{n}$, it follows from Hölder's inequality that

$$
\|A x\|_{\infty}=\max _{i=1, \ldots, m}\left|\operatorname{row}_{i}(A) x\right| \leq \max _{i=1, \ldots, m}\left\|\operatorname{row}_{i}(A)\right\|_{\bar{p}}\|x\|_{p},
$$

which implies that $\|A\|_{\infty, p} \leq \max _{i=1, \ldots, m}\left\|\operatorname{row}_{i}(A)\right\|_{\bar{p}}$. Next, let $j \in\{1, \ldots, n\}$ be such that $\left\|\operatorname{row}_{j}(A)\right\|_{\bar{p}}=\max _{i=1, \ldots, m}\left\|\operatorname{row}_{i}(A)\right\|_{\bar{p}}$ and let $x$ be such that $\left|\operatorname{row}_{j}(A) x\right|$ $=\left\|\operatorname{row}_{j}(A)\right\|_{\bar{p}}\|x\|_{p} . \quad$ Hence $\quad\|A x\|_{\infty}=\max _{i=1, \ldots, m}\left|\operatorname{row}_{i}(A) x\right| \geq\left|\operatorname{row}_{j}(A) x\right|=$ $\left\|\operatorname{row}_{j}(A)\right\|_{\bar{p}}\|x\|_{p}$ which implies that $\|A\|_{\infty, p} \geq \max _{i=1, \ldots, m}\left\|\operatorname{row}_{i}(A)\right\|_{\bar{p}}$ and hence (10) holds.

Remark 2.1. Note that (9) and (10) generalize the well-known expressions $\|A\|_{1,1}=\max _{i=1, \ldots, n}\left\|\operatorname{col}_{i}(A)\right\|_{1}[\mathrm{HJ}],\|A\|_{\infty, \infty}=\max _{i=1, \ldots, m}\left\|\operatorname{row}_{i}(A)\right\|_{1}[\mathrm{HJ}]$, and $\|A\|_{\infty, 1}=\|A\|_{\infty}[\mathrm{K}]$. Furthermore, since $\max _{i=1, \ldots, n}\left\|\operatorname{col}_{i}(A)\right\|_{2}=\mathrm{d}_{\max }^{1 / 2}\left(A^{\mathrm{T}} A\right)$ and $\max _{i=1, \ldots, m}\left\|\operatorname{row}_{i}(A)\right\|_{2}=\mathrm{d}_{\max }^{1 / 2}\left(A A^{\mathrm{T}}\right)$, it follows from (9) with $p=2$ that $\|A\|_{2,1}=\mathrm{d}_{\max }^{1 / 2}\left(A^{\mathrm{T}} A\right)$ and from (10) with $p=2$ that $\|A\|_{\infty, 2}=\mathrm{d}_{\max }^{1 / 2}\left(A A^{\mathrm{T}}\right)$.

Lemma 2.2. Let $\|\cdot\|^{\prime}$ and $\|\cdot\|^{\prime \prime}$ denote absolute vector norms on $\mathbb{R}^{n}$ and $\mathbb{R}^{m}$, respectively, and let $\|\cdot\|: \mathbb{R}^{m \times n} \rightarrow \mathbb{R}$ be the matrix norm induced by $\|\cdot\|^{\prime}$ and $\|\cdot\|^{\prime \prime}$. Then the following statements hold:

(i) Let $A \in \mathbb{R}^{m \times n}$ be such that $A \geq \geq 0$. Then there exists $x \in \mathbb{R}^{n}$ such that $x \geq \geq 0,\|x\|^{\prime}=1$, and $\|A\|=\|A x\|^{\prime \prime}$.

(ii) Let $A, B \in \mathbb{R}^{m \times n}$ be such that $0 \leq \leq A \leq \leq B$. Then $\|A\| \leq\|B\|$.

Proof. To prove (i) let $y \in \mathbb{R}^{n}$ be such that $\|y\|^{\prime}=1$ and $\|A\|=\|A y\|^{\prime \prime}$. Now, since $\|\cdot\|^{\prime}$ and $\|\cdot\|^{\prime \prime}$ are absolute (and hence monotone) vector norms it follows that

$$
\|A\|=\|A y\|^{\prime \prime} \leq\|A|y|\|^{\prime \prime} \leq\|A\|\||y|\|^{\prime}=\|A\|\|y\|^{\prime}=\|A\|,
$$

which implies that $\|A\|=\|A|y|\|^{\prime \prime}$ and hence (i) follows with $x=|y|$.

Next, to prove (ii) let $x \in \mathbb{R}^{n}$ be such that $x \geq \geq 0,\|x\|^{\prime}=1$, and $\|A\|=\|A x\|^{\prime \prime}$ (the existence of such an $x$ follows from (i)). Hence, since $\|\cdot\|^{\prime \prime}$ is an absolute vector norm and $A x \leq \leq B x$ it follows that

$$
\|A\|=\|A x\|^{\prime \prime} \leq\|B x\|^{\prime \prime} \leq\|B\|,
$$

which implies (ii).

The following result generalizes Hölder's inequality to mixed-signal norms.

Lemma 2.3. Let $p, r \in[1, \infty]$, and let $f \in \mathrm{L}_{p}$ and $g \in \mathrm{L}_{\bar{p}}$. Then

$$
\langle f, g\rangle \leq\left|\left\|f\left|\|\|_{p, r}\right|\right\| g \|_{\bar{p}, \bar{r}} .\right.
$$

Finally, the following two results are needed for the results given in Section 3. 
Lemma 2.4 [W2]. Let $p \in[1, \infty)$ and $r \in[1, \infty]$, and let $f \in \mathrm{L}_{p}$. Then

$$
\left.|||f|\right|_{p, r}=\sup _{g \in \mathscr{G}}\langle f, g\rangle \text {, }
$$

where $\mathscr{G} \triangleq\left\{g \in \mathrm{L}_{\bar{p}}:\||| g \mid\|_{\bar{p}, \bar{r}} \leq 1\right\}$.

Lemma 2.5 [W2]. Let $p \in[1, \infty), r \in[1, \infty]$, and $f:[0, \infty) \times[0, \infty) \rightarrow \mathbb{R}^{n}$ be such that $f(t, \cdot)$ is integrable for almost all $t \in[0, \infty), f(\cdot, \tau) \in \mathrm{L}_{p}$ for almost all $\tau \in[0, \infty)$, and $g \in \mathrm{L}_{1}$, where $g(\tau) \triangleq\left[\int_{0}^{\infty}\|f(t, \tau)\|_{r}^{p} \mathrm{~d} t\right]^{1 / p}$. Then

$$
\left|\|y \mid\|_{p, r} \leq \int_{0}^{\infty} g(\tau) \mathrm{d} \tau,\right.
$$

where

$$
y(t)=\int_{0}^{\infty} f(t, \tau) \mathrm{d} \tau, \quad t \geq 0
$$

\section{Induced Convolution Operator Norms for Linear Systems}

In this section we develop induced convolution operator norms. For the system (1), (2), let $G: \mathbb{R} \rightarrow \mathbb{R}^{l \times m}$ denote the impulse response function

$$
G(t) \triangleq \begin{cases}0, & t<0, \\ C e^{A t} B, & t \geq 0 .\end{cases}
$$

Next, let $\mathcal{G}: \mathrm{L}_{p} \rightarrow \mathrm{L}_{q}$ denote the convolution operator

$$
y(t)=(\mathcal{G} * u)(t) \triangleq \int_{0}^{\infty} G(t-\tau) u(\tau) \mathrm{d} \tau,
$$

and define the induced norm $\|\mathcal{G}\| \|_{(q, s),(p, r)}$ as

$$
\|\mathcal{G}\|_{(q, s),(p, r)} \triangleq \sup _{\|u\|_{p, r}=1}\|\mathcal{G} * u\| \|_{q, s} .
$$

The following lemma provides an explicit expression for $\|\mathcal{G}\|_{(\infty, \infty),(r, r)}$ for the case in which $\mathcal{G}$ is a single-input/single-output operator.

Lemma 3.1. Let $r \in[1, \infty]$ and let $l=m=1$. Then $\mathcal{G}: \mathrm{L}_{r} \rightarrow \mathrm{L}_{\infty}$, and

$$
\|\mathcal{G}\|_{(\infty, \infty),(r, r)}=\||| G \mid\|_{\bar{r}, \bar{r}} \text {. }
$$

Proof. For $r=1$ and $r=\infty,(18)$ is standard; see [W2] and [DV, pp. 23-24], respectively. Next, let $r \in(1, \infty)$ and note, for all $t \geq 0$, it follows from Lemma 2.3 with $p=r$ that

$$
\begin{aligned}
|y(t)| & =\left|\int_{0}^{\infty} G(t-\tau) u(\tau) \mathrm{d} \tau\right| \leq\left.\left[\int_{0}^{\infty}|G(t-\tau)|^{\bar{r}} \mathrm{~d} \tau\right]^{1 / \bar{r}}\|u\|\right|_{r, r} \\
& =\left[\int_{0}^{t}|G(\tau)|^{\bar{r}} \mathrm{~d} \tau\right]^{1 / \bar{r}}\left|\left\|u \left|\left\|\left.\right|_{r, r} \leq\left|\left\|G \left|\left\|_{\bar{r}, \bar{r}}|||u|\right\|_{r, r}\right.\right.\right.\right.\right.\right.\right.
\end{aligned}
$$


which implies

$$
\|\mathcal{G}\|_{(\infty, \infty),(r, r)} \leq\|\| G \mid \|_{\bar{r}, \bar{r}} .
$$

Next, let $T>0$ and let $u(\cdot)$ be such that $u(t)=\operatorname{sgn}(G(T-t))|G(T-t)|^{1 /(r-1)}$, $t \geq 0$, where $\operatorname{sgn}(\cdot)$ denotes the signum function. Now, since $\|u\| \|_{r, r}=$ $\left[\int_{0}^{\infty}|G(T-t)|^{\bar{r}} \mathrm{~d} t\right]^{1 / r}$, it follows that

$$
\begin{aligned}
|y(T)| & =\left|\int_{0}^{\infty} G(T-\tau) u(\tau) \mathrm{d} \tau\right|=\int_{0}^{\infty}|G(T-\tau)|^{\bar{r}} \mathrm{~d} \tau \\
& =\left[\int_{0}^{\infty}|G(T-\tau)|^{\bar{r}} \mathrm{~d} \tau\right]^{1 / \bar{r}}\left|\left\|u||_{r, r}=\left[\int_{0}^{T}|G(\tau)|^{\bar{r}} \mathrm{~d} \tau\right]^{1 / \bar{r}} \mid\right\| u\|\|_{r, r}\right.
\end{aligned}
$$

Hence,

$$
\||y|\|_{\infty, \infty} \geq \lim _{T \rightarrow \infty}|y(T)|=\left|\left\|G \left|\left\|_{\bar{r}, \bar{r}}|||u|\right\|_{r, r}\right.\right.\right.
$$

which, with (19), implies (18).

Remark 3.1. Note that it follows from Lemma 3.1 that there exists $u \in \mathrm{L}_{r}$ such that $\lim _{t \rightarrow \infty}(\mathcal{G} * u)(t)=\left.\left.\|\| G||\right|_{\bar{r}, \bar{r}}|| u||\right|_{r, r}$.

Remark 3.2. Lemma 3.1 can also be derived using the concept of interpolation spaces [BL]. Specifically, it follows from the Riesz-Thorin interpolation theorem [BL, p. 2] that if $\mathcal{G}: \mathrm{L}_{\infty} \rightarrow \mathrm{L}_{\infty}$ and $\mathcal{G}: \mathrm{L}_{1} \rightarrow \mathrm{L}_{\infty}$, then $\mathcal{G}: \mathrm{L}_{r} \rightarrow \mathrm{L}_{\infty}$ for all $r \in$ $(1, \infty)$.

Next define $\mathcal{P} \in \mathbb{R}^{m \times m}$ and $\mathcal{Q} \in \mathbb{R}^{l \times l}$ by

$$
\mathcal{P} \triangleq \int_{0}^{\infty} G^{\mathrm{T}}(t) G(t) \mathrm{d} t, \quad \mathcal{Q} \triangleq \int_{0}^{\infty} G(t) G^{\mathrm{T}}(t) \mathrm{d} t .
$$

Note that $\mathcal{P}=B^{\mathrm{T}} P B$ and $\mathcal{Q}=C Q C^{\mathrm{T}}$, where the observability and controllability Gramians $P$ and $Q$, respectively, are the unique $n \times n$ nonnegative-definite solutions to the Lyapunov equations

$$
0=A^{\mathrm{T}} P+P A+C^{\mathrm{T}} C, \quad 0=A Q+Q A^{\mathrm{T}}+B B^{\mathrm{T}} .
$$

Furthermore, let $G_{[p, q]}$ denote the $l \times m$ matrix whose $(i, j)$ th element is $\|\left.\left|G_{(i, j)}\right|\right|_{(p, p),(q, q)}$.

Theorem 3.1. The following statements hold:

(i) $\mathcal{G}: \mathrm{L}_{2} \rightarrow \mathrm{L}_{2}$, and

$$
\|\mathcal{G}\|_{(2,2),(2,2)}=\sup _{\omega \in \mathbb{R}} \sigma_{\max }(H(\jmath \omega)) .
$$

(ii) Let $r \in[1, \infty]$. Then $\mathcal{G}: \mathrm{L}_{1} \rightarrow \mathrm{L}_{2}$, and

$$
\|\mathcal{G}\|_{(2,2),(1, r)}=\left\|\mathcal{P}^{1 / 2}\right\|_{2, r} .
$$

(iii) Let $p \in[1, \infty]$. Then $\mathcal{G}: \mathrm{L}_{2} \rightarrow \mathrm{L}_{\infty}$, and

$$
\|\mid \mathcal{G}\|_{(\infty, p),(2,2)}=\left\|\mathcal{Q}^{1 / 2}\right\|_{2, \bar{p}} .
$$


(iv) Let $p, r \in[1, \infty]$. Then $\mathcal{G}: \mathrm{L}_{1} \rightarrow \mathrm{L}_{\infty}$, and

$$
\||\mathcal{G}|\|_{(\infty, p),(1, r)}=\sup _{t \geq 0}\|G(t)\|_{p, r} .
$$

(v) Let $r \in[1, \infty]$. Then $\mathcal{G}: \mathrm{L}_{r} \rightarrow \mathrm{L}_{\infty}$, and

$$
\|\mid \mathcal{G}\|_{(\infty, \infty),(r, r)}=\max _{i=1, \ldots, l}\left\|\operatorname{row}_{i}\left(G_{[\bar{r}, \bar{r}]}\right)\right\|_{\bar{r}} .
$$

(vi) Let $p \in[1, \infty]$. Then $\mathcal{G}: \mathrm{L}_{1} \rightarrow \mathrm{L}_{p}$, and

$$
\left\|\left.|\mathcal{G}|\right|_{(p, p),(1,1)}=\max _{j=1, \ldots, m}\right\| \operatorname{col}_{j}\left(G_{[p, p]}\right) \|_{p} .
$$

Proof. (i) is standard; see $[\mathrm{Z}]$ and $[\mathrm{F}]$ for a proof.

(ii) It follows from Lemma 2.5 that

$$
\begin{aligned}
\|y\|_{2,2} & \leq \int_{0}^{\infty}\|G(t-\tau) u(\tau)\|_{2,2} \mathrm{~d} \tau \\
& =\int_{0}^{\infty}\left\{\int_{0}^{\infty} u^{\mathrm{T}}(\tau) G^{\mathrm{T}}(t-\tau) G(t-\tau) u(\tau) \mathrm{d} t\right\}^{1 / 2} \mathrm{~d} \tau \\
& =\int_{0}^{\infty}\left\|\mathcal{P}^{1 / 2} u(\tau)\right\|_{2} \mathrm{~d} \tau \leq \int_{0}^{\infty}\left\|\mathcal{P}^{1 / 2}\right\|_{2, r}\|u(\tau)\|_{r} \mathrm{~d} \tau=\left\|\mathcal{P}^{1 / 2}\right\|_{2, r}\|u\| \|_{1, r},
\end{aligned}
$$

which implies that $\|\mid \mathcal{G}\|_{(2,2),(1, r)} \leq\left\|\mathcal{P}^{1 / 2}\right\|_{2, r}$.

Next, let $u_{k}(\cdot)=\hat{u} v_{k}(\cdot), k=1,2, \ldots$, where $\hat{u} \in \mathbb{R}^{d}$ is such that $\|\hat{u}\|_{r}=1$, $\left\|\mathcal{P}^{1 / 2} \hat{u}\right\|_{2}=\left\|\mathcal{P}^{1 / 2}\right\|_{2, r}\|\hat{u}\|_{r}$, and measurable $v_{k}:[0, \infty) \rightarrow \mathbb{R}$ is such that $\left\|\left|v_{k}\right|\right\|_{1,1}$ $=1$ and, as $k \rightarrow \infty, v_{k}(\cdot) \rightarrow \delta(\cdot)$, where $\delta(\cdot)$ is the Dirac delta function. Note that $\left\|\left|u_{k}\right|\right\|_{1, r}=1, k=1,2, \ldots$, and $y_{k}(t) \rightarrow G(t) \hat{u}, t \geq 0$, as $k \rightarrow \infty$, where $y_{k}(t) \triangleq$ $\left(\mathcal{G} * u_{k}\right)(t)$. Hence,

$$
\begin{aligned}
\|\mathcal{G}\|_{(2,2),(1, r)} & \geq \lim _{k \rightarrow \infty}\left\|\mid y_{k}\right\|_{2,2}=\left\{\int_{0}^{\infty}\|G(t) \hat{u}\|_{2}^{2} \mathrm{~d} t\right\}^{1 / 2}=\left\{\int_{0}^{\infty} \hat{u}^{\mathrm{T}} G^{\mathrm{T}}(t) G(t) \hat{u} \mathrm{~d} t\right\}^{1 / 2} \\
& =\left(\hat{u}^{\mathrm{T}} \mathcal{P} \hat{u}\right)^{1 / 2}=\left\|\mathcal{P}^{1 / 2} \hat{u}\right\|_{2}=\left\|\mathcal{P}^{1 / 2}\right\|_{2, r},
\end{aligned}
$$

which implies that $\|\mid \mathcal{G}\|_{(2,2),(1, r)}=\left\|\mathcal{P}^{1 / 2}\right\|_{2, r}$.

(iii) With $p=r=2$ it follows from Lemma 2.3 that, for all $t \geq 0$,

$$
\begin{aligned}
\|y(t)\|_{p} & =\left\|\int_{0}^{\infty} G(t-\tau) u(\tau) \mathrm{d} \tau\right\|_{p}=\max _{\left\{\hat{u} \in \mathbb{R}^{n}:\|\| \hat{u} \|_{p}=1\right\}} \int_{0}^{\infty} \hat{u}^{\mathrm{T}} G(t-\tau) u(\tau) \mathrm{d} \tau \\
& \leq \max _{\left\{\hat{u} \in \mathbb{R}^{n}:\|\hat{u}\|_{p}=1\right\}}\left[\int_{0}^{\infty}\|G(t-\tau) \hat{u}\|_{2}^{2} \mathrm{~d} \tau\right]^{1 / 2}\|u\|_{2,2} \\
& =\max _{\left\{\hat{u} \in \mathbb{R}^{n}:\|\hat{u}\|_{p}=1\right\}}\left[\hat{u}^{\mathrm{T}} \int_{0}^{t} G(\tau) G^{\mathrm{T}}(\tau) \mathrm{d} \tau \hat{u}\right]^{1 / 2} \mid\|u\|_{2,2} \\
& \leq \max _{\left\{\hat{u} \in \mathbb{R}^{n}:\|\hat{u}\|_{p}=1\right\}}\left(\hat{u}^{\mathrm{T}} \mathcal{Q} \hat{u}\right)^{1 / 2}\|\| u\left\|_{2,2}=\right\| \mathcal{Q}^{1 / 2}\left\|_{2, \bar{p}}\right\| u\|\|_{2,2},
\end{aligned}
$$


which implies that $\left|\left\|y\left|\left\|_{\infty, p} \leq\right\| \mathcal{Q}^{1 / 2}\left\|_{2, \bar{p}} \mid\right\| u \|_{2,2}\right.\right.\right.$ for all $y \in \mathrm{L}_{\infty}$ and $u \in \mathrm{L}_{2}$, and hence $\|\mid \mathcal{G}\|_{(\infty, p),(2,2)} \leq\left\|\mathcal{Q}^{1 / 2}\right\|_{2, \bar{p}}$.

Next, let $\hat{u} \in \mathbb{R}^{d}$ be such that $\|\hat{u}\|_{\bar{p}}=1$ and $\left\|\mathcal{Q}^{1 / 2} \hat{u}\right\|_{2}=\left\|\mathcal{Q}^{1 / 2}\right\|_{2, \bar{p}}$, and let $T>0$ and

$$
u(t)=\frac{1}{\left\|\mathcal{Q}^{1 / 2}\right\|_{2, \bar{p}}} G^{\mathrm{T}}(T-t) \hat{u},
$$

so that $\|\mid u\|_{2,2} \leq 1$. Now, since $\|\cdot\|_{p \mathrm{D}}=\|\cdot\|_{\bar{p}}$, it follows that

$$
\begin{aligned}
\|y \mid\|_{\infty, p} & =\sup _{t \geq 0}\|y(t)\|_{p} \\
& =\sup _{t \geq 0} \max _{\left\{\hat{y} \in \mathbb{R}^{n}:\|\hat{y}\|_{p}=1\right\}} \hat{y}^{\mathrm{T}} y(t) \\
& \geq \sup _{t \geq 0} \hat{u}^{\mathrm{T}} y(t) \\
& =\frac{1}{\left\|\mathcal{Q}^{1 / 2}\right\|_{2, \bar{p}}} \sup _{t \geq 0} \int_{0}^{\infty} \hat{u}^{\mathrm{T}} G(T-\tau) G^{\mathrm{T}}(T-\tau) \hat{u} \mathrm{~d} \tau \\
& =\frac{1}{\left\|\mathcal{Q}^{1 / 2}\right\|_{2, \bar{p}}} \sup _{t \geq 0} \int_{0}^{T} \hat{u}^{\mathrm{T}} G(\tau) G^{\mathrm{T}}(\tau) \hat{u} \mathrm{~d} \tau,
\end{aligned}
$$

which implies that, for every $T>0$, there exists $u \in \mathrm{L}_{2}$ such that $\|u\| \|_{2,2} \leq 1$ and

$$
\||y|\|_{\infty, p} \geq \frac{1}{\left\|\mathcal{Q}^{1 / 2}\right\|_{2, \bar{p}}} \sup _{t \geq 0} \int_{0}^{T} \hat{u}^{\mathrm{T}} G(\tau) G^{\mathrm{T}}(\tau) \hat{u} \mathrm{~d} \tau,
$$

or, equivalently,

$$
\begin{aligned}
\|\mathcal{G}\|_{(\infty, p),(2,2)} & \geq \sup _{T>0} \frac{1}{\left\|\mathcal{Q}^{1 / 2}\right\|_{2, \bar{p}}} \sup _{t \geq 0} \int_{0}^{T} \hat{u}^{\mathrm{T}} G(\tau) G^{\mathrm{T}}(\tau) \hat{u} \mathrm{~d} \tau \\
& =\frac{1}{\left\|\mathcal{Q}^{1 / 2}\right\|_{2, \bar{p}}} \sup _{t \geq 0} \int_{0}^{\infty} \hat{u}^{\mathrm{T}} G(\tau) G^{\mathrm{T}}(\tau) \hat{u} \mathrm{~d} \tau \\
& =\frac{1}{\left\|\mathcal{Q}^{1 / 2}\right\|_{2, \bar{p}}} \hat{u}^{\mathrm{T}} \mathcal{Q} \hat{u} \\
& =\left\|\mathcal{Q}^{1 / 2}\right\|_{2, \bar{p}},
\end{aligned}
$$

which further implies that $\|\mathcal{G}\|_{(\infty, p),(2,2)}=\left\|\mathcal{Q}^{1 / 2}\right\|_{2, \bar{p}}$.

(iv) Note that, for all $t \geq 0$,

$$
\begin{aligned}
\|y(t)\|_{p} & \leq \int_{0}^{\infty}\|G(t-\tau) u(\tau)\|_{p} \mathrm{~d} \tau \leq \int_{0}^{\infty}\|G(t-\tau)\|_{p, r}\|u(\tau)\|_{r} \mathrm{~d} \tau \\
& \leq \sup _{t \geq 0}\|G(t)\|_{p, r} \int_{0}^{\infty}\|u(\tau)\|_{r} \mathrm{~d} \tau \leq \sup _{t \geq 0}\|G(t)\|_{p, r}\|\| u \|_{1, r},
\end{aligned}
$$

which implies that $\|\mid \mathcal{G}\|_{(\infty, p),(1, r)} \leq \sup _{t \geq 0}\|G(t)\|_{p, r}$. 
Next, let $\varepsilon>0$ and $t_{0} \in[0, \infty)$ be such that $\left\|G\left(t_{0}\right)\right\|_{p, r}>\sup _{t \geq 0}\|G(t)\|_{p, r}-\varepsilon$. In addition, let $u_{k}(\cdot)=v_{k}(\cdot) \hat{u}, k=1,2, \ldots$, where $\hat{u} \in \mathbb{R}^{n}$ is such that $\|\hat{u}\|_{r}=1$, $\left\|G\left(t_{0}\right) \hat{u}\right\|_{p}=\left\|G\left(t_{0}\right)\right\|_{p, r}\|\hat{u}\|_{r}$, and measurable $v_{k}:[0, \infty) \rightarrow \mathbb{R}$ is such that $\left\|v_{k} \mid\right\|_{1,1}$ $=1$ and, as $k \rightarrow \infty, v_{k}(\cdot) \rightarrow \delta(\cdot)$, where $\delta(\cdot)$ is the Dirac delta function. In this case note that $\left\|\left|u_{k}\right|\right\|_{1, r}=1, k=1,2, \ldots$, and $y_{k}(t) \rightarrow G(t) \hat{u}, t \geq 0$, as $k \rightarrow \infty$, where $y_{k}(t) \triangleq\left(\mathcal{G} * u_{k}\right)(t)$. Hence,

$$
\begin{aligned}
\|\mathcal{G}\|_{(\infty, p),(1, r)} & \geq \lim _{k \rightarrow \infty} \sup _{t \geq 0}\left\|y_{k}(t)\right\|_{p}=\sup _{t \geq 0}\|G(t) \hat{u}\|_{p} \\
& \geq\left\|G\left(t_{0}\right) \hat{u}\right\|_{p}=\left\|G\left(t_{0}\right)\right\|_{p, r}>\sup _{t \geq 0}\|G(t)\|_{p, r}-\varepsilon
\end{aligned}
$$

which implies that

$$
\sup _{t \geq 0}\|G(t)\|_{p, r}-\varepsilon<\|\| \mathcal{G}\left\|_{(\infty, p),(1, r)} \leq \sup _{t \geq 0}\right\| G(t) \|_{p, r}, \quad \varepsilon>0,
$$

and hence (25) holds.

(v) Note that for all $u \in \mathrm{L}_{r}$ and $y \in \mathrm{L}_{\infty}$ it follows that $\|u\|\left\|_{r, r}=\right\| \bar{u} \|_{r}$ and $\|y \mid\|_{\infty, \infty}=\|\bar{y}\|_{\infty}$, where $\bar{u} \in \mathbb{R}^{m}$ and $\bar{y} \in \mathbb{R}^{l}$ with $\bar{u}_{i}=\left\|u_{i} \mid\right\| \|_{r, r}, i=1, \ldots, m$, and $\bar{y}_{i}=\|\| y_{i}\|\|_{\infty, \infty}, \quad i=1, \ldots, l$. Next, it follows from Lemma 3.1 that $\left\||| \mathcal{G}_{(i, j)}\right\|_{(\infty, \infty),(r, r)}=||\left|G_{(i, j)}\right| \|_{\bar{r}, \bar{r}}$ and hence

$$
\begin{aligned}
\left\|y_{i}\right\| \|_{\infty, \infty} & =\left\|\left|\sum_{j=1}^{m} \mathcal{G}_{(i, j)} * u_{j}\left\|\left.\right|_{\infty, \infty} \leq \sum_{j=1}^{m}\right\| \mathcal{G}_{(i, j)} * u_{j}\|\|_{\infty, \infty}\right.\right. \\
& \leq \sum_{j=1}^{m}\left\|\left|\mathcal{G}_{(i, j)}\right|\right\|\left\|_{\bar{r}, \bar{r}}\right\| u_{j}\|\|_{r, r} \leq\left\|\operatorname{row}_{i}\left(G_{[\bar{r}, \bar{r}]}\right)\right\|_{\bar{r}}\|\bar{u}\|_{r} \\
& \leq \max _{i=1, \ldots, l}\left\|\operatorname{row}_{i}\left(G_{[\bar{r}, \bar{r}]}\right)\right\|_{\bar{r}}\|\bar{u}\|_{r},
\end{aligned}
$$

which implies that $\||| y \mid\|_{\infty, \infty}=\|\bar{y}\|_{\infty} \leq \max _{i=1, \ldots, l}\left\|\operatorname{row}_{i}\left(G_{[\bar{r}, \bar{r}]}\right)\right\|\left\|_{\bar{r}}\right\| u\|\|_{r, r}$ and hence,

$$
\|\mathcal{H}\|_{(\infty, \infty),(r, r)} \leq \max _{i=1, \ldots, l}\left\|\operatorname{row}_{i}\left(G_{[\bar{r}, \bar{r}]}\right)\right\|_{\bar{r}} .
$$

Next, let $I \in\{1, \ldots, l\}$ be such that $\left\|\operatorname{row}_{I}\left(G_{[\bar{r}, \bar{r}]}\right)\right\|_{\bar{r}}=\max _{i=1, \ldots, l}\left\|\operatorname{row}_{i}\left(G_{[\bar{r}, \bar{r}]}\right)\right\|_{\bar{r}}$. Now, let $\hat{u} \in \mathbb{R}^{m}$ be such that $\|\hat{u}\|_{r}=1$, let $\operatorname{row}_{I}\left(G_{[\bar{r}, \bar{r}]}\right) \hat{u}=\left\|\operatorname{row}_{I}\left(G_{[\bar{r}, \bar{r}]}\right)\right\|_{\vec{r}}$, and let $u_{j} \in \mathrm{L}_{r}, j=1, \ldots, m$, be such that $\left\|u_{j} \mid\right\|_{r, r}=\hat{u}_{j}$ and $\lim _{t \rightarrow \infty}\left(\mathcal{G}_{(I, j)} * u_{j}\right)(t)=$ ||| $G_{(I, j)}\left|\left\|_{\bar{r}, \bar{r}}||\left|u_{j}\right|\right\|_{r, r}\right.$. Note that the existence of such a $u_{j}(\cdot)$ follows from Lemma 3.1 and Remark 3.1. Now,

$$
\begin{aligned}
\|y \mid\|_{\infty, \infty} & \geq||\left|y_{I}\right| \|_{\infty, \infty} \geq \lim _{t \rightarrow \infty}\left|y_{I}(t)\right|=\lim _{t \rightarrow \infty}\left|\sum_{j=1}^{m}\left(\mathcal{G}_{(I, j)} * u_{j}\right)(t)\right| \\
& =\sum_{j=1}^{m}||\left|G_{(I, j)}\right|\|\|_{\bar{r}, \bar{r}}|| u_{j} \mid\left\|_{r, r}=\operatorname{row}_{I}\left(G_{[\bar{r}, \bar{r}]}\right) \bar{u}=\right\| \operatorname{row}_{I}\left(G_{[\bar{r}, \bar{r}]}\right) \|_{\bar{r}},
\end{aligned}
$$

which, with (28), implies (26). 
(vi) For $p=\infty,(27)$ is a direct consequence of (iv) or (v). Now, let $p \in[1, \infty)$ and note that it follows from Lemma 2.4 that $\||y|\|_{p, p}=\sup _{\left\{\hat{y} \in \mathrm{L}_{\tilde{p}}:\|\hat{y}\| \|_{\dot{p}, \hat{p}}=1\right\}}\langle y, \hat{y}\rangle$. Hence, with $p=r=1$ it follows from Lemma 2.3 that

$$
\begin{aligned}
\|y\|_{p, p} & =\sup _{\|\hat{y}\|_{\dot{p}, \dot{p}}=1} \int_{0}^{\infty} y^{\mathrm{T}}(t) \hat{y}(t) \mathrm{d} t \\
& =\sup _{\|\hat{y}\|_{\dot{p}, \dot{p}}=1} \int_{0}^{\infty}\left(\int_{0}^{\infty} u^{\mathrm{T}}(\tau) G^{\mathrm{T}}(t-\tau) \mathrm{d} \tau\right) \hat{y}(t) \mathrm{d} t \\
& =\sup _{\|\hat{y}\|_{\dot{p}, \dot{p}}=1} \int_{0}^{\infty} u^{\mathrm{T}}(\tau)\left(\int_{0}^{\infty} G^{\mathrm{T}}(t-\tau) \hat{y}(t) \mathrm{d} t\right) \mathrm{d} \tau \\
& =\sup _{\|\hat{y}\|_{\dot{p}, \hat{p}}=1}\langle u, \hat{u}\rangle \\
& \leq\|\| u\left\|_{1,1} \sup _{\|\hat{y}\| \|_{\dot{p}, \hat{p}}=1}\right\| \hat{u} \|_{\infty, \infty},
\end{aligned}
$$

where $\hat{u}(t) \triangleq \int_{0}^{\infty} G^{\mathrm{T}}(\tau-t) \hat{y}(\tau) \mathrm{d} \tau$. Now, with $r=\bar{p}$, it follows from $(\mathrm{v})$ that

$$
\|\mid \mathcal{G}\|_{(p, p),(1,1)} \leq \sup _{\|\hat{y}\| \|_{p, p}=1}\|\hat{u}\|_{\infty, \infty}=\max _{j=1, \ldots, m}\left\|\operatorname{col}_{j}\left(G_{[p, p]}\right)\right\|_{p} .
$$

Next, let $J \in\{1, \ldots, m\}$ be such that $\left\|\operatorname{col}_{J}\left(G_{[p, p]}\right)\right\|_{p}=\max _{j=1, \ldots, m}\left\|\operatorname{col}_{j}\left(G_{[p, p]}\right)\right\|_{p}$ and let $u_{k}(\cdot) \triangleq v_{k}(\cdot) e_{J}, k=1,2, \ldots$, where $v_{k}:[0, \infty) \rightarrow \mathbb{R}$ is a measurable function such that $\left\|v_{k} \mid\right\|_{1,1}=1$ and, as $k \rightarrow \infty, v_{k}(\cdot) \rightarrow \delta(\cdot)$, where $\delta(\cdot)$ is the Dirac delta function. In this case note that $\left\|u_{k}\right\|_{1,1}=1, k=1,2, \ldots$, and $y_{k}(t) \rightarrow$ $\operatorname{col}_{J}(G(t)), t \geq 0$, as $k \rightarrow \infty$, where $y_{k}(t) \triangleq\left(\mathcal{G} * u_{k}\right)(t)$. Hence,

$$
\begin{aligned}
\|\mathcal{G}\|_{(p, p),(1,1)} & \geq \lim _{k \rightarrow \infty}\left\|y_{k}\right\|\left\|_{p, p}=\right\| \mid \operatorname{col}_{J}(G)\|\|_{p, p} \\
& =\left\|\operatorname{col}_{J}\left(G_{[p, p]}\right)\right\|_{p}=\max _{j=1, \ldots, m}\left\|\operatorname{col}_{j}\left(G_{[p, p]}\right)\right\|_{p},
\end{aligned}
$$

which, with (29), implies (27).

The following corollary specializes Theorem 3.1 to the results given in [W2], [W3], and [DV, p. 26].

Corollary 3.1. The following statements hold:

(i) $\mathcal{G}: \mathrm{L}_{1} \rightarrow \mathrm{L}_{2},\|\mathcal{G}\|_{(2,2),(1,2)}=\sigma_{\max }^{1 / 2}(\mathcal{P})$, and $\|\mid \mathcal{G}\|_{(2,2),(1,1)}=\mathrm{d}_{\max }^{1 / 2}(\mathcal{P})$.

(ii) $\mathcal{G}: \mathrm{L}_{2} \rightarrow \mathrm{L}_{\infty},\|\| \mathcal{G} \|_{(\infty, 2),(2,2)}=\sigma_{\max }^{1 / 2}(\mathcal{Q})$, and \|\| $\mathcal{G} \|_{(\infty, \infty),(2,2)}=\mathrm{d}_{\max }^{1 / 2}(\mathcal{Q})$.

(iii) $\mathcal{G}: \mathrm{L}_{1} \rightarrow \mathrm{L}_{\infty}, \quad\|\mid\| \mathcal{G}\left\|_{(\infty, \infty),(1,1)}=\sup _{t \geq 0}\right\| G(t) \|_{\infty}, \quad$ and \|\| $\mathcal{G} \|_{(\infty, 2),(1,2)}=$ $\sup _{t \geq 0} \sigma_{\max }(G(t))$.

(iv) $\mathcal{G}: \mathrm{L}_{\infty} \rightarrow \mathrm{L}_{\infty}$, and $\|\mid \mathcal{G}\|_{(\infty, \infty),(\infty, \infty)}=\max _{i=1, \ldots, l}\left\|\operatorname{row}_{i}\left(G_{[1,1]}\right)\right\|_{1}$.

(v) $\mathcal{G}: \mathrm{L}_{1} \rightarrow \mathrm{L}_{1}$, and $\|\mid \mathcal{G}\|\left\|_{(1,1),(1,1)}=\max _{j=1, \ldots, m}\right\| \operatorname{col}_{j}\left(G_{[1,1]}\right) \|_{1}$.

Remark 3.3. Recall that the $\mathrm{H}_{2}$ norm of the system (1), (2) is given by $\|\mid \mathcal{G}\| \|_{\mathrm{H}_{2}}$ $=\left\|\mathcal{P}^{1 / 2}\right\|_{\mathrm{F}}=\left\|\mathcal{Q}^{1 / 2}\right\|_{\mathrm{F}}$. Hence, using the fact that $\|\cdot\|_{\mathrm{F}}=\sigma_{\max }(\cdot)$ for rank-one 
matrices, it follows from (i) of Corollary 3.1 that if $B$ (and hence $\mathcal{P}$ ) is a rank-one matrix, then $\|\mid \mathcal{G}\|_{\mathrm{H}_{2}}=\|\| \mathcal{G} \|_{(2,2),(1,2)}$. Similarly, it follows from (iii) of Corollary 3.1 that if $C$ (and hence $\mathcal{Q}$ ) is a rank-one matrix, then $\|\mathcal{G}\|_{\mathrm{H}_{2}}=\|\mathcal{H} \mid\|_{(\infty, 2),(2,2)}$. Hence, in the single-input/multi-output and multi-output/single-input cases the $\mathrm{H}_{2}$ norm of a dynamical system is induced. In the multi-input/multi-output case, however, the $\mathrm{H}_{2}$ norm does not appear to be induced. For related details see [CH1].

Remark 3.4. Theorem 3.1 also applies to the more general case where $\mathcal{G}$ is a noncausal, time-invariant operator. In this case, the input-output spaces $\mathrm{L}_{p}$ and $\mathrm{L}_{r}$ are defined for $t \in(-\infty, \infty), H(\jmath \omega)$ is the Fourier transform of $G(t)$, and the lower limit in the integrals defining $\mathcal{P}$ and $\mathcal{Q}$ is replaced by $-\infty$.

Remark 3.5. Theorem 3.1 along with the Riesz-Thorin interpolation theorem [BL, p. 2] can be used to obtain a whole class of induced operator norm bounds. To see this, let $p_{0}, q_{0}, p_{1}, q_{1} \in[1, \infty]$, let $\theta \in(0,1)$, and define $p$ and $q$ by

$$
\frac{1}{p}=\frac{1-\theta}{p_{0}}+\frac{\theta}{p_{1}}
$$

and

$$
\frac{1}{q}=\frac{1-\theta}{q_{0}}+\frac{\theta}{q_{1}} .
$$

Furthermore, let $\||\mathcal{G}|\|_{0}$ denote an induced norm of $\mathcal{G}: \mathrm{L}_{p_{0}} \rightarrow \mathrm{L}_{q_{0}}$ and let $\|\mathcal{G} \mid\|_{1}$ denote an induced norm of $\mathcal{G}: \mathrm{L}_{p_{1}} \rightarrow \mathrm{L}_{q_{1}}$. Now, it follows from the Riesz-Thorin interpolation theorem [BL, p. 2] that if $\mathcal{G}: \mathrm{L}_{p_{0}} \rightarrow \mathrm{L}_{q_{0}}$ and $\mathcal{G}: \mathrm{L}_{p_{1}} \rightarrow \mathrm{L}_{q_{1}}$, then $\mathcal{G}: \mathrm{L}_{p} \rightarrow \mathrm{L}_{q}$. In addition, if $\|\mathcal{G}\|||$ denotes an induced norm of $\mathcal{G}: \mathrm{L}_{p} \rightarrow \mathrm{L}_{q}$, then

$$
\left\|\left|\mathcal { G } \left\|\left|\leq\|\mathcal{G}\|_{0}^{1-\theta}\||\mathcal{G}|\|_{1}^{\theta} .\right.\right.\right.\right.
$$

Using this result and Theorem 3.1, a wide class of induced operator norm bounds can be derived. For example, if $\mathcal{G}: \mathrm{L}_{2} \rightarrow \mathrm{L}_{2}$ and $\mathcal{G}: \mathrm{L}_{2} \rightarrow \mathrm{L}_{\infty}$, with $p_{0}=p_{1}=2$, $q_{0}=2$, and $q_{1}=\infty$, it follows that $\mathcal{G}: \mathrm{L}_{2} \rightarrow \mathrm{L}_{q}$ for all $q \in(2, \infty)$. In addition,

$$
\begin{aligned}
\|\mathcal{G}\|_{(q, 2),(2,2)} & \leq\|\mathcal{G}\|_{(2,2),(2,2)}^{2 / q}\|\mathcal{G}\|_{(\infty, 2),(2,2)}^{q-2 / q} \\
& =\sup _{\omega \in \mathbb{R}} \sigma_{\max }(H(\jmath \omega))^{2 / q}\left(\sigma_{\max }(\mathcal{Q})\right)^{q-2 / 2 q} .
\end{aligned}
$$

Remark 3.6. An analogous theorem to Theorem 3.1 can also be derived for discrete-time linear systems. Specifically, if the input-output spaces are replaced by the sequence spaces $\ell_{p}$ and $\ell_{r}$, respectively, $H(\jmath \omega), \omega \in \mathbb{R}$, and $G(t), t \geq 0$, are replaced by $H\left(e^{\jmath \omega}\right), \omega \in[0,2 \pi]$, and $G(k), k=0,1, \ldots$, respectively, and $\mathcal{P}$ and $\mathcal{Q}$ are replaced by

$$
\mathcal{P}=\sum_{k=0}^{\infty} G^{\mathrm{T}}(k) G(k), \quad \mathcal{Q}=\sum_{k=0}^{\infty} G(k) G^{\mathrm{T}}(k),
$$

where

$$
G(k) \triangleq \begin{cases}0, & k \leq 0 \\ C A^{k-1} B, & k>0\end{cases}
$$


then an analogous theorem to Theorem 3.1 holds for discrete-time systems. For specific details of this fact see [CHBW].

It is important to note that even though Theorem 3.1 is derived for linear, finite-dimensional dynamical systems, infinite-dimensional generalizations of this theorem can be readily developed. Specifically, let $x(t)$ in (1) be an element of a real separable Hilbert space $\mathcal{H}$ and assume that the state differential equation (1) is interpreted in the weak sense (see, e.g., p. 229 of [B]). Furthermore, assume the closed, densely defined operator $A: \mathcal{D}(A) \subset \mathcal{H} \rightarrow \mathcal{H}$ generates a strongly continuous semigroup $e^{A t}, t \geq 0$, on $\mathcal{H}$ and $A$ is exponentially stable. Finally, let $\mathcal{B}\left(\mathbb{R}^{m}, \mathcal{H}\right)$ denote the space of bounded linear operators from $\mathbb{R}^{m}$ into $\mathcal{H}$ and let $\mathcal{C}\left(\mathcal{H}, \mathbb{R}^{l}\right)$ denote the space of bounded linear operators from $\mathcal{H}$ into $\mathbb{R}^{l}$ so that $B \in \mathcal{B}\left(\mathbb{R}^{m}, \mathcal{H}\right)$ and $C \in \mathcal{C}\left(\mathcal{H}, \mathbb{R}^{l}\right)$. Now, if $\mathcal{H}$ is such that $G:[0, \infty) \rightarrow \mathbb{R}^{l \times m}$ and $\mathcal{P}$ and $\mathcal{Q}$ are well defined, then Theorem 3.1 is still valid. In this case, $\mathcal{P}=B^{*} P B$ and $\mathcal{Q}=C Q C^{*}$, where $(\cdot)^{*}$ denotes the adjoint operator, and $P$ and $Q$ are the unique solutions to the infinite-dimensional observability and controllability Gramians satisfying

$$
\begin{array}{ll}
0=A^{*} P x+P A x+C^{*} C x, & x \in \mathcal{D}(A), \\
0=A Q x+Q A^{*} x+B B^{*} x, & x \in \mathcal{D}\left(A^{*}\right) .
\end{array}
$$

The infinite-dimensional system description discussed above includes several classes of systems; namely, functional differential systems, delay systems, and systems described by irrational transfer functions. For an excellent textbook treatment of these systems the reader is referred to $[\mathrm{CZ}]$. Related works can also be found in $[\mathrm{M}]$, [W1], and [PU].

An alternative characterization of input-output properties is the Hankel norm which provides a mapping from past inputs $u(t), t \in(-\infty, 0]$, to future outputs $y(t), t \in[0, \infty)[\mathrm{G}],[\mathrm{W} 2]$. For causal dynamical systems the Hankel operator $\Gamma: \mathrm{L}_{p}(-\infty, 0] \rightarrow \mathrm{L}_{q}$ is defined by

$$
y(t)=(\Gamma * u)(t) \triangleq \int_{0}^{\infty} G(t+\tau) u(-\tau) \mathrm{d} \tau, \quad t \in[0, \infty),
$$

where $\mathrm{L}_{p}(-\infty, 0]$ denotes the set of functions in $\mathrm{L}_{p}$ on the time interval $(-\infty, 0]$, and the induced Hankel norm $\||\Gamma|\|_{(q, s),(p, r)}$ is defined by

$$
\||\Gamma|\|_{(q, s),(p, r)} \triangleq \sup _{\|u\| \|_{p, r}=1}\|\Gamma * u\| \|_{q, s} .
$$

Proposition 3.1. The following statements hold:

(i) $\Gamma: \mathrm{L}_{2}(-\infty, 0] \rightarrow \mathrm{L}_{2}$, and

$$
\||| \Gamma\|_{(2,2),(2,2)}=\lambda_{\max }^{1 / 2}(P Q) .
$$

(ii) Let $r \in[1, \infty]$. Then $\Gamma: \mathrm{L}_{1}(-\infty, 0] \rightarrow \mathrm{L}_{2}$, and

$$
\|\mid \Gamma\|_{(2,2),(1, r)}=\left\|\mathcal{P}^{1 / 2}\right\|_{2, r} .
$$


(iii) Let $p \in[1, \infty]$. Then $\Gamma: \mathrm{L}_{2}(-\infty, 0] \rightarrow \mathrm{L}_{\infty}$, and

$$
\|\mid \Gamma\|_{(\infty, p),(2,2)}=\left\|\mathcal{Q}^{1 / 2}\right\|_{2, \bar{p}} .
$$

(iv) Let $p, r \in[1, \infty]$. Then $\Gamma: \mathrm{L}_{1}(-\infty, 0] \rightarrow \mathrm{L}_{\infty}$, and

$$
\||\Gamma|\|_{(\infty, p),(1, r)}=\sup _{t \geq 0}\|G(t)\|_{p, r} .
$$

(v) Let $r \in[1, \infty]$. Then $\Gamma: \mathrm{L}_{r}(-\infty, 0] \rightarrow \mathrm{L}_{\infty}$, and

$$
\||\Gamma|\|_{(\infty, \infty),(r, r)}=\max _{i=1, \ldots, l}\left\|\operatorname{row}_{i}\left(G_{[\bar{r}, \bar{r}]}\right)\right\|_{\bar{r}} .
$$

(vi) Let $p \in[1, \infty]$. Then $\Gamma: \mathrm{L}_{1}(-\infty, 0] \rightarrow \mathrm{L}_{p}$, and

$$
\||\Gamma|\|_{(p, p),(1,1)}=\max _{j=1, \ldots, m}\left\|\operatorname{col}_{j}\left(G_{[p, p]}\right)\right\|_{p} .
$$

Proof. (i) is standard; see [G] for a proof. The proof of (ii)-(vi) is similar to that of (ii)-(vi) of Theorem 3.1 with appropriate modifications to the time interval for the input space.

\section{Finitely Computable Upper Bounds for $\||\mathcal{G}|\|_{(\infty, p),(1, r)}$}

In this section we obtain a finitely computable upper bound for (25). To do this we assume that there exist $H_{\mathcal{L}}(s), H_{\mathcal{R}}(s) \in \mathcal{R} \mathrm{H}_{2}$ such that $H(s)=H_{\mathcal{L}}(s) H_{\mathcal{R}}(s)$, where $H(s) \in \mathcal{R} \mathrm{H}_{2}$ denotes the Laplace transform of $G(t)$. Note that such a factorization exists only if $H(s)$ has relative degree two. Furthermore, note that the above factorization exists if and only if there exist linear, time-invariant asymptotically stable dynamical systems with impulse response functions $G_{\mathcal{L}}: \mathbb{R} \rightarrow$ $\mathbb{R}^{l \times m_{1}}$ and $G_{\mathcal{R}}: \mathbb{R} \rightarrow \mathbb{R}^{m_{1} \times m}$ such that $G_{\mathcal{L}}(t)=0$ and $G_{\mathcal{R}}(t)=0, t<0$, and

$$
G(t)=\int_{0}^{\infty} G_{\mathcal{L}}(t-\tau) G_{\mathcal{R}}(\tau) \mathrm{d} \tau, \quad t \geq 0
$$

Next, let $\mathcal{G}_{\mathcal{L}}: \mathrm{L}_{2} \rightarrow \mathrm{L}_{\infty}$ and $\mathcal{G}_{\mathcal{R}}: \mathrm{L}_{1} \rightarrow \mathrm{L}_{2}$ denote the convolution operators of $G_{\mathcal{L}}$ and $G_{\mathcal{R}}$, respectively, and define $\mathcal{P}_{\mathcal{R}} \in \mathbb{R}^{m \times m}$ and $\mathcal{Q}_{\mathcal{L}} \in \mathbb{R}^{l \times l}$ by

$$
\mathcal{P}_{\mathcal{R}} \triangleq \int_{0}^{\infty} G_{\mathcal{R}}^{\mathrm{T}}(t) G_{\mathcal{R}}(t) \mathrm{d} t, \quad \mathcal{Q}_{\mathcal{L}} \triangleq \int_{0}^{\infty} G_{\mathcal{L}}(t) G_{\mathcal{L}}^{\mathrm{T}}(t) \mathrm{d} t .
$$

Finally, let $G_{\mathcal{L}}(t)=C_{\mathcal{L}} e^{A_{\mathcal{L}} t} B_{\mathcal{L}}, t \geq 0$, and $G_{\mathcal{R}}(t)=C_{\mathcal{R}} e^{A_{\mathcal{R}} t} B_{\mathcal{R}}, t \geq 0$, where $A_{\mathcal{L}} \in$ $\mathbb{R}^{n_{1} \times n_{1}}, B_{\mathcal{L}} \in \mathbb{R}^{n_{1} \times m_{1}}, C_{\mathcal{L}} \in \mathbb{R}^{l \times n_{1}}, A_{\mathcal{R}} \in \mathbb{R}^{n_{\mathrm{r}} \times n_{\mathrm{r}}}, B_{\mathcal{R}} \in \mathbb{R}^{n_{\mathrm{r}} \times m}$, and $C_{\mathcal{R}} \in \mathbb{R}^{m_{1} \times n_{\mathrm{r}}}$, and let $P_{\mathcal{R}} \in \mathbb{R}^{n_{\mathrm{r}} \times n_{\mathrm{r}}}$ and $Q_{\mathcal{L}} \in \mathbb{R}^{n_{1} \times n_{1}}$ be the unique, nonnegative definite solutions to the Lyapunov equations

$$
0=A_{\mathcal{R}}^{\mathrm{T}} P_{\mathcal{R}}+P_{\mathcal{R}} A_{\mathcal{R}}+C_{\mathcal{R}}^{\mathrm{T}} C_{\mathcal{R}}, \quad 0=A_{\mathcal{L}} Q_{\mathcal{L}}+Q_{\mathcal{L}} A_{\mathcal{L}}^{\mathrm{T}}+B_{\mathcal{L}} B_{\mathcal{L}}^{\mathrm{T}}
$$

Note that $\mathcal{P}_{\mathcal{R}}=B_{\mathcal{R}}^{\mathrm{T}} P_{\mathcal{R}} B_{\mathcal{R}}$ and $\mathcal{Q}_{\mathcal{L}}=C_{\mathcal{L}} Q_{\mathcal{L}} C_{\mathcal{L}}^{\mathrm{T}}$. 
Proposition 4.1. Let $p, r \in[1, \infty]$. If there exist $G_{\mathcal{L}}: \mathbb{R} \rightarrow \mathbb{R}^{l \times m_{1}}$ and $G_{\mathcal{R}}: \mathbb{R} \rightarrow$ $\mathbb{R}^{m_{1} \times m}$ such that (41) holds, then

$$
\|\mid \mathcal{G}\|_{(\infty, p),(1, r)} \leq\left\|\mathcal{Q}_{\mathcal{L}}^{1 / 2}\right\|_{2, \bar{p}}\left\|\mathcal{P}_{\mathcal{R}}^{1 / 2}\right\|_{2, r} .
$$

Proof. Note that $y(t)=\left(\mathcal{G}_{\mathcal{L}} *\left(\mathcal{G}_{\mathcal{R}} * u\right)\right)(t)$. Now, since $\mathcal{G}_{\mathcal{L}}: \mathrm{L}_{2} \rightarrow \mathrm{L}_{\infty}$ and $\mathcal{G}_{\mathcal{R}}$ : $\mathrm{L}_{1} \rightarrow \mathrm{L}_{2}$ it follows from Theorem 3.1 that

$$
\|y\|_{\infty, p} \leq\left\|\mathcal{Q}_{\mathcal{L}}^{1 / 2}\right\|_{2, \bar{p}} \mid\left\|\mathcal{G}_{\mathcal{R}} * u\right\|_{2,2} \leq\left\|\mathcal{Q}_{\mathcal{L}}^{1 / 2}\right\|_{2, \bar{p}}\left\|\mathcal{P}_{\mathcal{R}}^{1 / 2}\right\|_{2, r}\|u\|_{1, r}
$$

which implies (44).

The following corollary to Proposition 4.1 provides finitely computable bounds for the mixed-induced signal norm (25).

Corollary 4.1. Let $\mathcal{P}_{\mathcal{R}}$ and $\mathcal{Q}_{\mathcal{L}}$ be given by (42). Then the following inequalities hold:

(i) \|\| $\mathcal{G} \|_{(\infty, \infty),(1,1)} \leq \mathrm{d}_{\max }^{1 / 2}\left(\mathcal{Q}_{\mathcal{L}}\right) \mathrm{d}_{\max }^{1 / 2}\left(\mathcal{P}_{\mathcal{R}}\right)$.

(ii) \|\| $\mathcal{G} \|_{(\infty, 2),(1,2)} \leq \sigma_{\max }^{1 / 2}\left(\mathcal{Q}_{\mathcal{L}}\right) \sigma_{\max }^{1 / 2}\left(\mathcal{P}_{\mathcal{R}}\right)$.

(iii) \|\| $\mathcal{G} \|_{(\infty, \infty),(1,2)} \leq \mathrm{d}_{\max }^{1 / 2}\left(\mathcal{Q}_{\mathcal{L}}\right) \sigma_{\max }^{1 / 2}\left(\mathcal{P}_{\mathcal{R}}\right)$.

(iv) $\|\mid \mathcal{G}\|_{(\infty, 2),(1,1)} \leq \sigma_{\max }^{1 / 2}\left(\mathcal{Q}_{\mathcal{L}}\right) \mathrm{d}_{\max }^{1 / 2}\left(\mathcal{P}_{\mathcal{R}}\right)$.

Proof. The results follow from Theorem 3.1 and Proposition 4.1.

\section{Upper Bounds for $L_{1}$ Operator Norms}

In this section we provide upper bounds for the $\mathrm{L}_{1}$ operator norm $\|\mathcal{H}\|_{(\infty, p),(\infty, r)}$. For $\alpha>0$, define the shifted impulse response function $G_{\alpha}: \mathbb{R} \rightarrow \mathbb{R}^{l \times m}$ by

$$
G_{\alpha}(t) \triangleq \begin{cases}0 & t<0, \\ C e^{(A+(\alpha / 2) I) t} B, & t \geq 0,\end{cases}
$$

and let $\mathcal{G}_{\alpha}$ denote its convolution operator

$$
y(t)=\left(\mathcal{G}_{\alpha} * u\right)(t) \triangleq \int_{0}^{\infty} G_{\alpha}(t-\tau) u(\tau) \mathrm{d} \tau .
$$

Furthermore, for some of the results in this section we assume there exist $H_{\mathcal{L}_{\alpha}}(s), H_{\mathcal{R}_{\alpha}}(s) \in \mathcal{R} \mathrm{H}_{2} \quad$ such that $H_{\alpha}(s)=H_{\mathcal{L}_{\alpha}}(s) H_{\mathcal{R}_{\alpha}}(s)$, where $H_{\alpha}(s) \in \mathcal{R} \mathrm{H}_{2}$ denotes the Laplace transform of $G_{\alpha}(t)$. Note that the above factorization exists if and only if there exist linear time-invariant asymptotically stable dynamical systems with impulse response functions $G_{\mathcal{L}_{\alpha}}: \mathbb{R} \rightarrow \mathbb{R}^{l \times m_{1}}$ and $G_{\mathcal{R}_{\alpha}}: \mathbb{R} \rightarrow \mathbb{R}^{m_{1} \times m}$ such that $G_{\mathcal{L}_{\alpha}}(t)=0$ and $G_{\mathcal{R}_{\alpha}}(t)=0, t<0$, and

$$
G_{\alpha}(t)=\int_{0}^{\infty} G_{\mathcal{L}_{\alpha}}(t-\tau) G_{\mathcal{R}_{\alpha}}(\tau) \mathrm{d} \tau, \quad t \geq 0 .
$$

Next, let $\mathcal{G}_{\mathcal{L}_{\alpha}}: \mathrm{L}_{2} \rightarrow \mathrm{L}_{\infty}$ and $\mathcal{G}_{\mathcal{R}_{\alpha}}: \mathrm{L}_{1} \rightarrow \mathrm{L}_{2}$ denote the convolution operators of 
$G_{\mathcal{L}_{\alpha}}$ and $G_{\mathcal{R}_{\alpha}}$, respectively, and define $\mathcal{P}_{\mathcal{R}_{\alpha}} \in \mathbb{R}^{m \times m}$ and $\mathcal{Q}_{\mathcal{L}_{\alpha}} \in \mathbb{R}^{l \times l}$ by

$$
\mathcal{P}_{\mathcal{R}_{\alpha}} \triangleq \int_{0}^{\infty} G_{\mathcal{R}_{\alpha}}^{\mathrm{T}}(t) G_{\mathcal{R}_{\alpha}}(t) \mathrm{d} t, \quad \mathcal{Q}_{\mathcal{L}_{\alpha}} \triangleq \int_{0}^{\infty} G_{\mathcal{L}_{\alpha}}(t) G_{\mathcal{L}_{\alpha}}^{\mathrm{T}}(t) \mathrm{d} t
$$

Theorem 5.1. Let $\alpha>0$ be such that $A+(\alpha / 2) I$ is asymptotically stable and let $Q_{\alpha} \in \mathbb{R}^{n \times n}$ be the unique, nonnegative definite solution to the Lyapunov equation

$$
0=A Q_{\alpha}+Q_{\alpha} A^{\mathrm{T}}+\alpha Q_{\alpha}+B B^{\mathrm{T}} .
$$

Furthermore, let $p, r \in[1, \infty]$. Then $\mathcal{G}: \mathrm{L}_{\infty} \rightarrow \mathrm{L}_{\infty}$,

$$
\left\|\left|\mathcal{G}\left\|_{(\infty, p),(\infty, 2)} \leq \frac{1}{\sqrt{\alpha}}\right\| \mathcal{G}_{\alpha}\left\|\left.\right|_{(\infty, p),(2,2)}=\frac{1}{\sqrt{\alpha}}\right\|\left(C Q_{\alpha} C^{\mathrm{T}}\right)^{1 / 2} \|_{2, \bar{p}},\right.\right.
$$

and

$$
\|\mid \mathcal{G}\|_{(\infty, p),(\infty, r)} \leq \frac{2}{\alpha}\left\|\mathcal{G}_{\alpha}\right\|_{(\infty, p),(1, r)}=\frac{2}{\alpha} \sup _{t \geq 0}\left\|G_{\alpha}(t)\right\|_{p, r} .
$$

In addition, if there exist $G_{\mathcal{L}_{\alpha}}: \mathbb{R} \rightarrow \mathbb{R}^{l \times m_{1}}$ and $G_{\mathcal{R}_{\alpha}}: \mathbb{R} \rightarrow \mathbb{R}^{m_{1} \times m}$ such that (47) holds, then

$$
\|\mid \mathcal{G}\|_{(\infty, p),(\infty, r)} \leq \frac{2}{\alpha}\left\|\mathcal{G}_{\alpha}\right\|\left\|_{(\infty, p),(1, r)} \leq \frac{2}{\alpha}\right\| \mathcal{Q}_{\mathcal{L}_{\alpha}}^{1 / 2}\left\|_{2, \bar{p}}\right\| \mathcal{P}_{\mathcal{R}_{\alpha}}^{1 / 2} \|_{2, r} .
$$

Proof. Let $T>0, u \in \mathrm{L}_{\infty}$, and define

$$
u_{T}(t) \triangleq \begin{cases}e^{(\alpha / 2)(t-T)} u(t), & 0 \leq t \leq T \\ 0, & t>T .\end{cases}
$$

Now, note that

$$
\begin{aligned}
\left\|u_{T} \mid\right\|_{2,2}^{2} & =\int_{0}^{\infty}\left\|u_{T}(t)\right\|_{2}^{2} \mathrm{~d} t=\int_{0}^{T} e^{\alpha(t-T)}\|u(t)\|_{2}^{2} \mathrm{~d} t \\
& \leq\|u\|\left\|_{\infty, 2}^{2} \int_{0}^{T} e^{\alpha(t-T)} \mathrm{d} t=\frac{1}{\alpha}\right\|\|u\|_{\infty, 2}^{2},
\end{aligned}
$$

or, equivalently, $\left\||| u_{T}\left|\left\|_{2,2} \leq(1 / \sqrt{\alpha})|| u \mid\right\|_{\infty, 2}\right.\right.$. Next, define $y_{T}(t) \triangleq e^{(\alpha / 2)(t-T)} y(t)$ and, since $G(t)=0, t<0$, note that

$$
\begin{aligned}
y_{T}(t) & =\int_{0}^{\infty} e^{(\alpha / 2)(t-T)} G(t-\tau) u(\tau) \mathrm{d} \tau=\int_{0}^{\infty} e^{(\alpha / 2)(t-\tau)} G(t-\tau) e^{(\alpha / 2)(\tau-T)} u(\tau) \mathrm{d} \tau \\
& =\int_{0}^{\infty} G_{\alpha}(t-\tau) u_{T}(\tau) \mathrm{d} \tau=\left(\mathcal{G}_{\alpha} * u_{T}\right)(t) .
\end{aligned}
$$

Next, it follows from (24) that

$$
\left\|y_{T}(t)\right\|_{p} \leq\left\||| \mathcal{G}_{\alpha}\left|\left\|\left.\right|_{(\infty, p),(2,2)}||\left|u_{T}\right|\right\|_{2,2} \leq \frac{1}{\sqrt{\alpha}}\right|\right\| \mathcal{G}_{\alpha}\left|\left\|_ { ( \infty , p ) , ( 2 , 2 ) } \left|\|u \mid\|_{\infty, 2} .\right.\right.\right.
$$


Now, noting that $y(T)=y_{T}(T)$ it follows that

$$
\|y(T)\|_{p} \leq \frac{1}{\sqrt{\alpha}}\left\|\mathcal{G}_{\alpha}\right\|_{(\infty, p),(2,2)} \mid\|u\|_{\infty, 2}, \quad T \geq 0,
$$

which implies (50).

Let $T>0, u \in \mathrm{L}_{\infty}$, and let $u_{T}(\cdot)$ be given by (53). Then

$$
\begin{aligned}
\|\| u_{T}\|\|_{1, r} & =\int_{0}^{\infty}\left\|u_{T}(t)\right\|_{r} \mathrm{~d} t=\int_{0}^{T} e^{(\alpha / 2)(t-T)}\|u(t)\|_{r} \mathrm{~d} t \\
& \leq\|\| u\|\|_{\infty, r} \int_{0}^{T} e^{(\alpha / 2)(t-T)} \mathrm{d} t=\frac{2}{\alpha}\|u\| \|_{\infty, r} .
\end{aligned}
$$

Now, it follows from (25) that

$$
\left\|y_{T}(t)\right\|_{p} \leq\left\|\left|\mathcal{G}_{\alpha}\right|\right\|_{(\infty, p),(1, r)}\left|\left\|u_{T}||_{1, r} \leq \frac{2}{\alpha}\right\|\right| \mathcal{G}_{\alpha}\left|\left\|_{(\infty, p),(1, r)} \mid\right\| u\|\|_{\infty, r} .\right.
$$

Hence, since $y(T)=y_{T}(T)$,

$$
\|y(T)\|_{p} \leq \frac{2}{\alpha}\left\|\mid \mathcal{G}_{\alpha}\right\|\left\|_{(\infty, p),(1, r)}\right\|\|u\| \|_{\infty, r}, \quad T \geq 0,
$$

which implies (51). Finally, (52) follows from (51) and Proposition 4.1.

Next we specialize Theorem 5.1 to Euclidean and infinity spatial norms.

Corollary 5.1. Let $\alpha>0$ be such that $A+(\alpha / 2) I$ is asymptotically stable, let $G_{\alpha}(\cdot)$ be given by (45), and let $Q_{\alpha} \in \mathbb{R}^{n \times n}$ be the unique, nonnegative definite solution to (49). Then the following statements hold:

$$
\|\mid \mathcal{G}\|_{(\infty, 2),(\infty, 2)} \leq \frac{1}{\sqrt{\alpha}}\left\|\mathcal{G}_{\alpha}\right\|_{(\infty, 2),(2,2)}=\frac{1}{\sqrt{\alpha}} \sigma_{\max }^{1 / 2}\left(C Q_{\alpha} C^{\mathrm{T}}\right) .
$$

Furthermore, if there exist $G_{\mathcal{L}_{\alpha}}: \mathbb{R} \rightarrow \mathbb{R}^{l \times m_{1}}$ and $G_{\mathcal{R}_{\alpha}}: \mathbb{R} \rightarrow \mathbb{R}^{m_{1} \times m}$ such that (47) holds, then

$$
\left\|\left|\mathcal{G}\left\|_{(\infty, 2),(\infty, 2)} \leq \frac{2}{\alpha}\right\|\right| \mathcal{G}_{\alpha}||_{(\infty, 2),(1,2)} \leq \frac{2}{\alpha} \sigma_{\max }^{1 / 2}\left(\mathcal{Q}_{\mathcal{L}_{\alpha}}\right) \sigma_{\max }^{1 / 2}\left(\mathcal{P}_{\mathcal{R}_{\alpha}}\right) .\right.
$$

(ii) $\quad\left\|\left|\mathcal{G}\left\|_{(\infty, \infty),(\infty, 2)} \leq \frac{1}{\sqrt{\alpha}}\right\|\right| \mathcal{G}_{\alpha}\right\|_{(\infty, \infty),(2,2)}=\frac{1}{\sqrt{\alpha}} \mathrm{d}_{\max }^{1 / 2}\left(C Q_{\alpha} C^{\mathrm{T}}\right)$.

Furthermore, if there exist $G_{\mathcal{L}_{\alpha}}: \mathbb{R} \rightarrow \mathbb{R}^{l \times m_{1}}$ and $G_{\mathcal{R}_{\alpha}}: \mathbb{R} \rightarrow \mathbb{R}^{m_{1} \times m}$ such that (47) holds, then

$$
\left\|\left|\mathcal{G}\left\|_{(\infty, \infty),(\infty, 2)} \leq \frac{2}{\alpha}\right\|\right| \mathcal{G}_{\alpha}\right\|_{(\infty, \infty),(1,2)} \leq \frac{2}{\alpha} \mathrm{d}_{\max }^{1 / 2}\left(\mathcal{Q}_{\mathcal{L}}\right) \sigma_{\max }^{1 / 2}\left(\mathcal{P}_{\mathcal{R}}\right) .
$$

Proof. The proof is a direct consequence of Lemma 2.1 and Theorem 5.1.

Remark 5.1. Using set theoretic arguments involving closed convex sets and support functions the $\mathrm{L}_{1}$ norm bound in (54) was given by Schweppe [S]. Within 
the context of $\mathrm{L}_{\infty}$ equi-induced norms, this $\mathrm{L}_{1}$ norm bound is referred to as the star-norm in [NAP] and [SHB]. The expression given by (55) provides an alternative finitely computable bound for the $\mathrm{L}_{\infty}$ equi-induced norm.

Next, we present an example to provide comparisons between the bounds (50)(52) given in Theorem 5.1 for $\|\mathcal{G}\| \|_{(\infty, p),(\infty, r)}, p, r \in[1, \infty]$.

Example 5.1. Consider the system (1), (2) with

$$
A=\left[\begin{array}{cc}
-1 & 0 \\
1 & -1
\end{array}\right], \quad B=\left[\begin{array}{l}
1 \\
0
\end{array}\right], \quad C=\left[\begin{array}{ll}
0 & 1
\end{array}\right],
$$

so that $G(t)=t e^{-t}, t \geq 0$. Since $\mathcal{G}$ is a convolution operator for a single-input/ single-output system it follows that $\|\mathcal{G}\|_{(\infty, \infty),(\infty, \infty)}=\|\| \mathcal{G} \|_{(\infty, p),(\infty, r)}, \quad p$, $r \in[1, \infty]$. Hence, it follows from Lemma 3.1 with $r=\infty$ that

$$
\left\|\left|\mathcal{G} \|_{(\infty, \infty),(\infty, \infty)}=\int_{0}^{\infty}\right| G(t) \mid \mathrm{d} t=\int_{0}^{\infty} t e^{-t} \mathrm{~d} t=1 .\right.
$$

Now, with $p=2, \quad$ it follows from $(50)$ that $\|\mid \mathcal{G}\|_{(\infty, \infty),(\infty, \infty)} \leq$ $(1 / \sqrt{\alpha})\left\|\left|\mathcal{G}_{\alpha}\right|\right\|_{(\infty, 2),(2,2)}$ for all $0<\alpha<2$. Noting that

$$
\left\|\mathcal{G}_{\alpha}\right\|_{(\infty, 2),(2,2)}^{2}=\int_{0}^{\infty} G_{\alpha}^{2}(t) \mathrm{d} t=\int_{0}^{\infty} t^{2} e^{-(2-\alpha) t} \mathrm{~d} t=\frac{2}{(2-\alpha)^{3}},
$$

it follows that

$$
\|\mid \mathcal{G}\|_{(\infty, \infty),(\infty, \infty)} \leq \inf _{0<\alpha<2} \frac{2}{\sqrt{\alpha(2-\alpha)^{3}}}=\sqrt{\frac{32}{27}} \approx 1.0887 .
$$

Next, using (51) to bound $\|\mathcal{G}\|_{(\infty, \infty),(\infty, \infty)}$ yields

$$
\|\mathcal{G}\|_{(\infty, \infty),(\infty, \infty)} \leq \frac{2}{\alpha} \sup _{t \geq 0} G_{\alpha}(t)=\frac{4}{\alpha(2-\alpha)} e^{-1}, \quad 0<\alpha<2,
$$

which implies

$$
\|\mid \mathcal{G}\|_{(\infty, \infty),(\infty, \infty)} \leq \inf _{0<\alpha<2} \frac{4}{\alpha(2-\alpha)} e^{-1}=4 e^{-1} \approx 1.4715 .
$$

Finally, we compare (52) with (50) and (51). Since $H_{\alpha}(s)=1 /(s+1-\alpha / 2)^{2}$ it follows from (52) with $H_{\mathcal{L}_{\alpha}}(s)=H_{\mathcal{R}_{\alpha}}(s)=1 /(s+1-\alpha / 2)$ that

$$
\||\mathcal{G}|\|_{(\infty, \infty),(\infty, \infty)} \leq \frac{2}{\alpha}\left\|\left|\mathcal { G } _ { \mathcal { L } _ { \alpha } } \left\|| _ { ( \infty , 2 ) , ( 2 , 2 ) } \left|\left\|\mathcal{G}_{\mathcal{R}_{\alpha}} \mid\right\|_{(2,2),(1,2)} .\right.\right.\right.\right.
$$

Hence,

$$
\|\mid \mathcal{G}\|_{(\infty, \infty),(\infty, \infty)} \leq \frac{2}{\alpha} \int_{0}^{\infty} e^{-(2-\alpha) t} \mathrm{~d} t=\frac{2}{\alpha(2-\alpha)}, \quad 0<\alpha<2,
$$


Table 1. Summary of induced operator norms for $p, r \in[1, \infty]$.

\begin{tabular}{|c|c|c|c|}
\hline Input & Output & Induced norm & Upper bound \\
\hline$\||| \cdot||_{2,2}$ & $\||\cdot|\|_{2,2}$ & $\sup _{\omega \in \mathbb{R}} \sigma_{\max }(H(\jmath \omega))$ & \\
\hline$\||\cdot|\|_{1, r}$ & $\mid\|\cdot \cdot\|_{2,2}$ & $\left\|\mathcal{P}^{1 / 2}\right\|_{2, r}$ & \\
\hline$\||| \cdot\|_{2,2}$ & \|\|$\cdot\|\|_{\infty, p}$ & $\left\|\mathcal{Q}^{1 / 2}\right\|_{2, \dot{p}}$ & \\
\hline$\||\cdot|\|_{1, r}$ & $\||\cdot|\|_{\infty, p}$ & $\sup _{t \geq 0}\|G(t)\|_{p, r}$ & $\left\|\mathcal{Q}_{\mathcal{L}}^{1 / 2}\right\|_{2, \bar{p}}\left\|\mathcal{P}_{\mathcal{R}}^{1 / 2}\right\|_{2, r}$ \\
\hline $\begin{array}{l}\|\| \cdot\|\|_{r, r} \\
\|\| \cdot \|_{1,1}\end{array}$ & \|\|$\cdot\|\|_{\infty, \infty}$ & $\max _{i=1, \ldots, l}\left\|\operatorname{row}_{i}\left(G_{[\dot{r}, \vec{r}]}\right)\right\|_{\dot{r}}$ & \\
\hline\|\|$\cdot\|\|_{\infty, 2}$ & \|\|$\cdot\|\|_{\infty, p}$ & 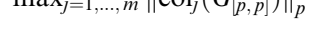 & $(1 / \sqrt{\alpha})\left\|C Q_{\alpha} C^{\mathrm{T}}\right\|_{2 \bar{p}}$ \\
\hline$\||| \cdot \mid\|_{\infty, r}$ & $\||| \cdot \mid\|_{\infty, p}$ & & $(2 / \alpha) \sup _{t \geq 0}\left\|G_{\alpha}(t)\right\|_{p, r}$ \\
\hline$\||| \cdot \mid\|_{\infty, r}$ & $\||| \cdot \mid\|_{\infty, p}$ & & $(2 / \alpha)\left\|\mathcal{Q}_{\mathcal{L}_{\alpha}}^{1 / 2}\right\|_{2, \bar{p}}\left\|\mathcal{P}_{\mathcal{R}_{\alpha}}^{1 / 2}\right\|_{2, r}$ \\
\hline
\end{tabular}

which implies

$$
\|\mathcal{G}\|_{(\infty, \infty),(\infty, \infty)} \leq \inf _{0<\alpha<2} \frac{2}{\alpha(2-\alpha)}=2 .
$$

A summary of the results of Sections $3-5$ is given in Table 1.

\section{Induced Convolution Operator Norms for Mixed-Norm Control}

In this section we apply the results of Section 3 to mixed-norm control problems. Specifically, we consider the performance analysis problem shown in Fig. 1, where $u_{i} \in \mathrm{L}_{p_{i}}, i=1, \ldots, l, y_{i} \in \mathrm{L}_{r_{i}}, i=1, \ldots, k, \mathcal{G}_{i j}: \mathrm{L}_{p_{j}} \rightarrow \mathrm{L}_{r_{i}}, i=1, \ldots, k, j=1, \ldots, l$, so that

$$
y_{i}(t)=\sum_{j=1}^{l}\left(\mathcal{G}_{i j} * u_{j}\right)(t),
$$

where $p_{i}, q_{i} \in[1, \infty], i=1, \ldots, l$, and $r_{i}, s_{i} \in[1, \infty], i=1, \ldots, k$, are given. Furthermore, let $G_{i j}: \mathbb{R} \rightarrow \mathbb{R}^{l_{i} \times m_{j}}, i=1, \ldots, k, j=1, \ldots, l$, denote the corresponding impulse response matrix function with $G_{i j}(t)=0, t<0, i=1, \ldots, k, j=1, \ldots, l$. Next, define $u(t) \triangleq\left[u_{1}^{\mathrm{T}}(t), \ldots, u_{l}^{\mathrm{T}}(t)\right]^{\mathrm{T}}, y(t) \triangleq\left[y_{1}^{\mathrm{T}}(t), \ldots, y_{k}^{\mathrm{T}}(t)\right]^{\mathrm{T}}$, and

$$
G(t)=\left[\begin{array}{c:c:c}
G_{11}(t) & \ldots & G_{1 l}(t) \\
\hdashline \vdots & \ddots & \vdots \\
\hdashline G_{k 1}(t) & \cdots & G_{k l}(\bar{t})
\end{array}\right]
$$

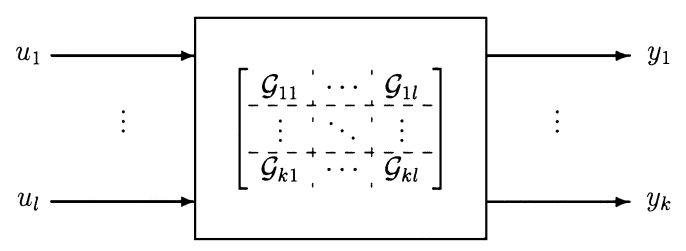

Fig. 1. Partitioned convolution operator. 
so that $y(t)=(\mathcal{G} * u)(t)$, where $u \in \mathcal{U} \triangleq \mathrm{L}_{p_{1}} \times \cdots \times \mathrm{L}_{p_{k}}$ and $y \in \mathcal{Y} \triangleq \mathrm{L}_{r_{1}} \times \cdots \times$ $\mathrm{L}_{r_{k}}$. Furthermore, for all $u \in \mathcal{U}$, define the corresponding block-norm vector $\bar{u} \in \mathbb{R}^{l}$ of $u(\cdot)$ by $\bar{u}_{i}=\left\|\left|u_{i}\right|\right\|_{p_{i}, q_{i}}, i=1, \ldots, l$, and, for all $y \in \mathcal{Y}$, define the corresponding block-norm vector $\bar{y} \in \mathbb{R}^{k}$ of $y(\cdot)$ by $\bar{y}_{i}=\|\| y_{i}\|\|_{r_{i}, s_{i}}, i=1, \ldots, k$. Next, we define an operator norm on $\mathcal{U}$ and $\mathcal{Y}$. Specifically, let $\|\cdot\|^{\prime}: \mathbb{R}^{l} \rightarrow \mathbb{R}$ and $\|\cdot\|^{\prime \prime}: \mathbb{R}^{k} \rightarrow \mathbb{R}$ be absolute vector norms and define the operator norms $\||\cdot|\|_{\mathcal{U}}: \mathcal{U} \rightarrow \mathbb{R}$ and $\left|\|\cdot \mid\|_{\mathcal{Y}}: \mathcal{Y} \rightarrow \mathbb{R}\right.$ by

$$
\left\|\left|\|u\|_{\mathcal{U}} \triangleq\|\bar{u}\|^{\prime}, \quad\|\mid y\|_{\mathcal{Y}} \triangleq\|\bar{y}\|^{\prime \prime} .\right.\right.
$$

In this case, the induced convolution operator norm $\||\mathcal{G}|\|_{\mathcal{Y}, \mathcal{U}}$ is defined as

$$
\||\mathcal{G}|\|_{\mathcal{Y}, \mathcal{U}} \triangleq \sup _{u \in \mathcal{U},\|u\|_{\mathcal{U}}=1}\|\mid \mathcal{G} * u\| \|_{\mathcal{Y}}
$$

The following result provides an upper bound to $\|\mathcal{G}\|_{\mathcal{Y}, \mathcal{U}}$. For the statement of this result define the block-norm matrix $\bar{G} \in \mathbb{R}^{k \times l}$ by

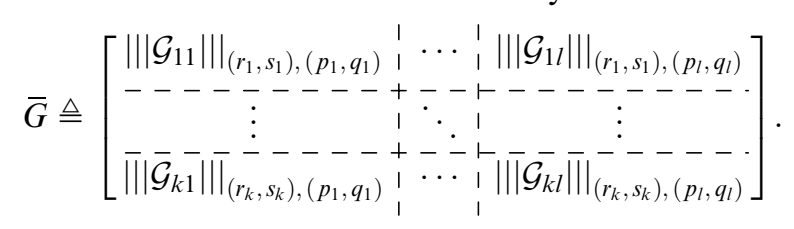

Theorem 6.1. The following statements hold:

(i) Let $\bar{G}$ be given by (62) and let $\tilde{G} \in \mathbb{R}^{k \times l}$ be such that $\bar{G} \leq \leq \tilde{G}$. Then

$$
\|\mathcal{G} \mid\|_{\mathcal{Y}, \mathcal{U}} \leq\|\bar{G}\| \leq\|\tilde{G}\|,
$$

where $\|\cdot\|$ denotes the matrix norm induced by $\|\cdot\|^{\prime}$ and $\|\cdot\|^{\prime \prime}$.

(ii) If $\|\cdot\|^{\prime}=\|\cdot\|_{1}$ and $\|\cdot\|^{\prime \prime}=\|\cdot\|_{\infty}$, then

$$
\|\mathcal{G}\|\left\|_{\mathcal{Y}, \mathcal{U}}=\right\| \bar{G} \|_{\infty}=\max _{\substack{i=1, \ldots, k \\ j=1, \ldots, l}} \bar{G}_{(i, j)} .
$$

Proof. To prove (i), note that, for all $i=1, \ldots, k$,

$\left.\left\|y_{i}\right\|\right|_{r_{i}, s_{i}}=\left.\left\||| \sum_{j=1}^{l} \mathcal{G}_{i j} * u_{j}\left|\left\|\left.\right|_{r_{i}, s_{i}} \leq \sum_{j=1}^{l}\right\|\right| \mathcal{G}_{i j} * u_{j}\right\|\right|_{r_{i}, s_{i}} \leq \sum_{j=1}^{l}\left\|\left|\mathcal{G}_{i j}\right|\right\|_{\left(r_{i}, s_{i}\right),\left(p_{j}, q_{j}\right)}\left\|\left|u_{j}\right|\right\|_{p_{j}, q_{j}}$,

which implies that $\bar{y} \leq \leq \bar{G} \bar{u}$. Hence, since $\|\cdot\|^{\prime \prime}$ is an absolute vector norm it follows that

$$
\left|\|y \mid\|_{\mathcal{Y}}=\|\bar{y}\|^{\prime \prime} \leq\|\bar{G} \bar{u}\|^{\prime \prime} \leq\|\bar{G}\|\|\bar{u}\|^{\prime}=\|\bar{G}\|\|\| u \|_{\mathcal{U}},\right.
$$

which implies that $\left\|\mathcal{G}_{\mid}\right\|_{\mathcal{Y}, \mathcal{U}} \leq\|\bar{G}\|$. Next, since $0 \leq \leq \bar{G} \leq \leq \tilde{G}$ it follows from Lemma 2.2 that $\|\bar{G}\| \leq\|\tilde{G}\|$ which proves (63).

Next, if $\|\cdot\|^{\prime}=\|\cdot\|_{1}$ and $\|\cdot\|^{\prime \prime}=\|\cdot\|_{\infty}$ it follows from (i) and Lemma 2.1 that

$$
\||\mathcal{G}|\|_{\mathcal{Y}, \mathcal{U}} \leq\|\bar{G}\|_{\infty, 1}=\|\bar{G}\|_{\infty} .
$$

Now, let $I \in\{1, \ldots, k\}$ and $J \in\{1, \ldots, l\}$ be such that $\bar{G}_{(I, J)}=\|\bar{G}\|_{\infty}$ and 
let $u_{J} \in \mathrm{L}_{p_{J}}$ be such that $\left\|\left|u_{J}\right|\right\|_{p_{J}, q_{J}}=1$ and $\bar{G}_{(I, J)}=\left\|\mathcal{G}_{I J} \mid\right\|_{\left(r_{I}, s_{I}\right),\left(p_{J}, q_{J}\right)}=$

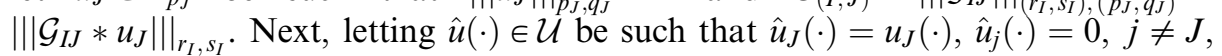
so that $y_{i}(t)=\left(\mathcal{G}_{i J} * u_{J}\right)(t), i=1, \ldots, k$, it follows that

$$
\||y|\|_{\mathcal{Y}}=\|\bar{y}\|_{\infty}=\max _{i=1, \ldots, k}\left\|\left|y_{i}\right|\right\|_{r_{i}, s_{i}} \geq\left\|\left|y_{I}\right|\right\|_{r_{I}, s_{I}}=\left\|\mathcal{G}_{I J} * u_{J}\right\| \|_{r_{I}, s_{I}}=\bar{G}_{(I, J)},
$$

which, noting ||$\hat{u}\left\|_{\mathcal{U}_{\mathcal{U}}}=\left.\left|\| u_{J}\right|\right|_{p_{J}, q_{J}}=1\right.$, yields $\| \mathcal{G} \mid\left\|_{\mathcal{Y}, \mathcal{U}} \geq\right\| \bar{G} \|_{\infty}$. Now, (64) follows from (65).

For a given dynamical system of the form (1), (2) with impulse response matrix function $G(\cdot)$ given by $(15)$ we write

$$
\mathcal{G} \sim\left[\begin{array}{l|l}
A & B \\
\hline C & 0
\end{array}\right]
$$

to denote the fact that the convolution operator $\mathcal{G}$ corresponds to the dynamical system (1), (2). The following corollary specializes Theorem 6.1 to the results given in [W3]. For the statement of this result let

$$
\mathcal{G}(\cdot)=\left[\begin{array}{ll}
\mathcal{G}_{11}(\cdot) & \mathcal{G}_{12}(\cdot) \\
\mathcal{G}_{21}(\cdot) & \mathcal{G}_{22}(\cdot)
\end{array}\right]
$$

where

$$
\begin{array}{ll}
\mathcal{G}_{11} \sim\left[\begin{array}{c|c}
A_{11} & B_{11} \\
\hline C_{11} & 0
\end{array}\right], & \mathcal{G}_{12} \sim\left[\begin{array}{c|c}
A_{12} & B_{12} \\
\hline C_{12} & 0
\end{array}\right], \\
\mathcal{G}_{21} \sim\left[\begin{array}{c|c}
A_{21} & B_{21} \\
\hline C_{21} & 0
\end{array}\right], & \mathcal{G}_{22} \sim\left[\begin{array}{c|c}
A_{22} & B_{22} \\
\hline C_{22} & 0
\end{array}\right] .
\end{array}
$$

Furthermore, let $P$ and $Q$ satisfy (21) with $(A, B, C)$ replaced by $\left(A_{21}, B_{21}, C_{21}\right)$ and $\left(A_{12}, B_{12}, C_{12}\right)$, respectively.

Corollary 6.1. The following statements hold:

(i) Let $\mathcal{U}=\mathrm{L}_{1} \times \mathrm{L}_{2}, \quad \mathcal{Y}=\mathrm{L}_{2}, \quad y(t)=\left(\mathcal{G}_{21} * u_{1}\right)(t)+\left(\mathcal{G}_{22} * u_{2}\right)(t), \quad\|u\|_{\mathcal{U}}=$ $\left|\left\|u_{1}\left|\left\|_{1, r}+||\left|u_{2}\right|\right\|_{2,2}\right.\right.\right.$, and $\left\||| y\left|\left\|_{\mathcal{Y}}=\left|\|y \mid\|_{2,2}\right.\right.\right.\right.$, where $r=1$ or 2 . Then

$$
\|\mid \mathcal{G}\|_{\mathcal{Y}, \mathcal{U}}=\max \left\{\left\|\left(B_{21}^{\mathrm{T}} P B_{21}\right)^{1 / 2}\right\|_{2, r}, \sup _{\omega \in \mathbb{R}} \sigma_{\max }\left(C_{22}\left(\jmath \omega I-A_{22}\right)^{-1} B_{22}\right)\right\} .
$$

(ii) Let $\mathcal{U}=\mathrm{L}_{2}, \quad \mathcal{Y}=\mathrm{L}_{\infty} \times \mathrm{L}_{2}, \quad y(t)=\left(\left[\mathcal{G}_{12}^{\mathrm{T}} \mathcal{G}_{22}^{\mathrm{T}}\right]^{\mathrm{T}} * u\right)(t), \quad\|u\|\left\|_{\mathcal{U}}=\right\|\|u\| \|_{2,2}$, and $\||| y \mid\|_{\mathcal{Y}}=\max \left\{||\left|y_{1}\right|\left\|_{\infty, p},||\left|y_{2}\right|\right\|_{2,2}\right\}$, where $p=2$ or $\infty$. Then

$$
\||\mathcal{G}|\|_{\mathcal{Y}, \mathcal{U}}=\max \left\{\left\|\left(C_{12} Q C_{12}^{\mathrm{T}}\right)^{1 / 2}\right\|_{2, q}, \sup _{\omega \in \mathbb{R}} \sigma_{\max }\left(C_{22}\left(\jmath \omega I-A_{22}\right)^{-1} B_{22}\right)\right\} .
$$

(iii) Let $\mathcal{U}=\mathrm{L}_{1} \times \mathrm{L}_{2}, \quad \mathcal{Y}=\mathrm{L}_{\infty} \times \mathrm{L}_{2}, \quad y(t)=(\mathcal{G} * u)(t), \quad\|u\|\left\|_{\mathcal{U}}=\right\|\left\|u_{1}\right\| \|_{1, r}+$ ||$\left|u_{2}\right|||_{2,2}$, and $\||| y \mid\|_{\mathcal{Y}}=\max \left\{||\left|y_{1}\right|||_{\infty, p},||\left|y_{2}\right| \|_{2,2}\right\}$, where $r=1$ or 2 and 


$$
\begin{aligned}
& p=2 \text { or } \infty \text {. Then } \\
& \|\mathcal{G}\| \|_{\mathcal{Y}, \mathcal{U}}=\max \left\{\left\|\left(C_{12} Q C_{12}^{\mathrm{T}}\right)^{1 / 2}\right\|_{2, q}, \sup _{\omega \in \mathbb{R}} \sigma_{\max }\left(C_{22}\left(\jmath \omega I-A_{22}\right)^{-1} B_{22}\right),\right. \\
& \left.\left\|\left(B_{21}^{\mathrm{T}} P B_{21}\right)^{1 / 2}\right\|_{2, r}, \sup _{t \geq 0}\left\|C_{11} e^{A_{11} t} B_{11}\right\|_{p, r}\right\} .
\end{aligned}
$$

Proof. The proof is a direct consequence of Theorem 6.1 using Theorem 3.1.

Finally, the following corollary to Theorem 6.1 provides a generalization of Corollary 6.1 to mixed-norm problems involving three-vector inputs and twovector outputs. For the statement of this result let

$$
\mathcal{G}(\cdot)=\left[\begin{array}{ccc}
\mathcal{G}_{11}(\cdot) & \mathcal{G}_{12}(\cdot) & \mathcal{G}_{13}(\cdot) \\
\mathcal{G}_{21}(\cdot) & \mathcal{G}_{22}(\cdot) & 0
\end{array}\right]
$$

where

$$
\begin{array}{rlrl}
\mathcal{G}_{11} \sim\left[\begin{array}{c|c}
A_{11} & B_{11} \\
\hline C_{11} & 0
\end{array}\right], & \mathcal{G}_{12} \sim\left[\begin{array}{c|c}
A_{12} & B_{12} \\
\hline C_{12} & 0
\end{array}\right], & \mathcal{G}_{21} \sim\left[\begin{array}{c|c}
A_{21} & B_{21} \\
\hline C_{21} & 0
\end{array}\right], \\
\mathcal{G}_{22} \sim\left[\begin{array}{c|c}
A_{22} & B_{22} \\
\hline C_{22} & 0
\end{array}\right], & \mathcal{G}_{13} \sim\left[\begin{array}{c|c}
A_{13} & B_{13} \\
\hline C_{13} & 0
\end{array}\right] .
\end{array}
$$

Furthermore, let $P$ and $Q$ satisfy (21) with $(A, B, C)$ replaced by $\left(A_{21}, B_{21}, C_{21}\right)$ and $\left(A_{12}, B_{12}, C_{12}\right)$, respectively.

Corollary 6.2. Let $\alpha>0, \mathcal{U}=\mathrm{L}_{1} \times \mathrm{L}_{2} \times \mathrm{L}_{\infty}, \mathcal{Y}=\mathrm{L}_{\infty} \times \mathrm{L}_{2}, \quad y(t)=(\mathcal{G} * u)(t)$, ||$|u|\left\|_{\mathcal{U}}=\left.||\left|u_{1}\right|\right|_{1, r}+||\left|u_{2}\right|\right\|_{2,2}+||\left|u_{3}\right|||_{\infty, r}$, and $\||y|\|_{\mathcal{Y}}=\max \left\{||\left|y_{1}\right|\left\|\left.\right|_{\infty, p},\right\|\left|y_{2}\right| \|_{2,2}\right\}$, where $r=1$ or 2 and $p=2$ or $\infty$. Then

$$
\begin{aligned}
\|\mathcal{G}\|_{\mathcal{Y}, \mathcal{U}} \leq \max \{ & \left\|\left(C_{12} Q C_{12}^{\mathrm{T}}\right)^{1 / 2}\right\|_{2, q}, \sup _{\omega \in \mathbb{R}} \sigma_{\max }\left(C_{22}\left(\jmath \omega I-A_{22}\right)^{-1} B_{22}\right), \\
& \left\|\left(B_{21}^{\mathrm{T}} P B_{21}\right)^{1 / 2}\right\|_{2, r}, \sup _{t \geq 0}\left\|C_{11} e^{A_{11} t} B_{11}\right\|_{p, r}, \\
& \left.\frac{2}{\alpha} \sup _{t \geq 0}\left\|C_{13} e^{\left(A_{13}+(\alpha / 2) I\right) t} B_{13}\right\|_{p, r}\right\} .
\end{aligned}
$$

Proof. The proof is a direct consequence of Theorem 6.1 using Theorems 3.1 and 5.1.

Remark 6.1. Note that the norms $\|\cdot\|^{\prime}$ and $\|\cdot\|^{\prime \prime}$ in Corollary 6.2 need not be restricted to $\|\cdot\|_{1}$ and $\|\cdot\|_{\infty}$, respectively. In this case upper bounds to $\|\mathcal{G}\|_{\mathcal{Y}, \mathcal{U}}$ can be obtained by computing $\|\tilde{G}\|$, where $\tilde{G}$ is given in Theorem 6.1 and $\|\cdot\|$ is the matrix norm induced by $\|\cdot\|^{\prime}$ and $\|\cdot\|^{\prime \prime}$. 


\section{Conclusion}

Explicit formulas for convolution operator norms and norm bounds induced by several classes of input-output signal pairs were derived. The results generalize previous system-norm results and can be used to capture disturbance rejection and robustness objectives for controlled dynamical systems.

\section{References}

[B] A. V. Balakrishnan, Applied Functional Analysis, Springer-Verlag, New York, 1981.

[BL] J. Bergh and J. Löfström, Interpolation Spaces, An Introduction, Springer-Verlag, Berlin, 1976.

[BH] D. S. Bernstein and W. M. Haddad, LQG control with an $\mathrm{H}_{\infty}$ performance bound: a Riccati equation approach, IEEE Trans. Automat. Control, 34 (1989), 293-305.

[CH1] V. Chellaboina and W. M. Haddad, Is the Frobenius matrix norm induced?, IEEE Trans. Automat. Control, 40 (1995), 2137-2139.

[CH2] V. Chellaboina and W. M. Haddad, Fixed-order dynamic compensation for linear systems with actuator amplitude and rate saturation constraints, Proc. Amer. Control Conf., Philadelphia, PA, 1998, pp. 3255-3259; also in Internat. J. Control, to appear.

[CHBW] V. Chellaboina, W. M. Haddad, D. S. Bernstein, and D. A. Wilson, Induced convolution operator norms for discrete-time linear systems, Proc. IEEE Conf. Dec. Control, Phoenix, AZ, 1999, pp. 487-492.

[CZ] R. F. Curtain and H. J. Zwart, An Introduction to Infinite-Dimensional Linear Systems Theory, Springer-Verlag, New York, 1995.

[DD] M. A. Dahleh and I. J. Diaz-Bobillo, Control of Uncertain Systems: A Linear Programming Approach, Prentice-Hall, Englewood Cliffs, NJ, 1995.

[DV] C. A. Desoer and M. Vidyasagar, Feedback Systems: Input-Output Properties, Academic Press, Orlando, FL, 1975.

[DEBW] S. G. Dharmasanam, R. S. Erwin, D. S. Bernstein, and D. A. Wilson, Synthesis of optimal generalized LQG and Hankel-norm controllers, Proc. Amer. Control Conf., Albuquerque, NM, 1997, pp. 3078-3082.

[F] B. A. Francis, A Course in $\mathrm{H}_{\infty}$ Control Theory, Springer-Verlag, New York, 1987.

[G] K. Glover, All optimal Hankel-norm approximations of linear multivariable systems and their $\mathrm{L}_{\infty}$-error bounds, Internat. J. Control, 39 (1984), 1115-1193.

[HJ] R. A. Horn and R. C. Johnson, Matrix Analysis, Cambridge University Press, Cambridge, 1985.

[K] W. Kahan, Numerical linear algebra, Canad. Math. Bull., 9 (1966), 757-801.

[M] I. J. Maddox, Infinite Matrices of Operators, Springer-Verlag, Berlin, 1980.

[NAP] K. Nagpal, J. Abedor, and K. Poolla, An LMI approach to peak-to-peak gain minimization: filtering and control, Internat. J. Robust Nonlinear Control, 6 (1996), 899-927.

[PU] J. R. Partington and B. Ünalmis, On the representation of shift-invariant operators by transfer functions, Systems Control Lett., 33 (1998), 25-30.

[R] M. A. Rotea, The generalized $\mathrm{H}_{2}$ control problem, Automatica, 29 (1993), 373-385.

[S] F. C. Schweppe, Uncertain Dynamic Systems, Prentice-Hall, Englewood Cliffs, NJ, 1973.

[SS] G. W. Stewart and J. Sun, Matrix Perturbation Theory, Academic Press, San Diego, CA, 1990.

[SHB] M. Sznaier, M. Holmes, and J. Bu, Mixed $\mathrm{H}_{2} / \mathrm{L}_{1}$ control with low order controllers: a linear matrix inequality approach, Proc. IEEE Conf. Dec. Control, New Orleans, LA, 1995, pp. $1352-1357$.

[V] M. Vidyasagar, Optimal rejection of persistent bounded disturbances, IEEE Trans. Automat. Control, 31 (1986), 527-535.

[W1] G. Weiss, Representation of shift-invariant operators on $\mathrm{L}_{2}$ by $\mathrm{H}_{\infty}$ transfer functions, Math. Control Signals Systems, 4 (1991), 193-203. 
[W2] D. A. Wilson, Convolution and Hankel operator norms of linear systems, IEEE Trans. Automat. Control, 34 (1989), 94-97.

[W3] D. A. Wilson, Induced norms for mixed $\mathrm{H}_{2} / \mathrm{H}_{\infty}$ and $\mathrm{H}_{2} / \mathrm{H}_{\infty} / \mathrm{L}_{\infty}$ control, Proc. IEEE Conf. Dec. Control, New Orleans, LA, 1995, pp. 3164-3168.

[WNH] D. A. Wilson, M. A. Nekou, and G. D. Halikias, An LQR weight selection approach to the discrete generalized $\mathrm{H}_{2}$ control problem, Internat. J. Control, 71 (1998), 93-101.

[Z] G. Zames, Feedback and optimal sensitivity: model reference transformations, multiplicative seminorms, and approximate inverses, IEEE Trans. Automat. Control, 26 (1981), 301-320.

[ZGBD] K. Zhou, K. Glover, B. Bodenheimer, and J. Doyle, Mixed $\mathrm{H}_{2}$ and $\mathrm{H}_{\infty}$ performance objectives, I: robust performance analysis, IEEE Trans. Automat. Control, 39 (1994), 1564-1574. 